\title{
THE COEVOLUTION OF NUCLEAR STAR CLUSTERS, MASSIVE BLACK HOLES, AND THEIR HOST GALAXIES
}

\author{
Fabio Antonini ${ }^{1}$, EnRico Barausse ${ }^{2,3}$, and Joseph Silk ${ }^{2,3,4,5}$ \\ ${ }^{1}$ Center for Interdisciplinary Exploration and Research in Astrophysics (CIERA) and Department of Physics and Astrophysics, \\ Northwestern University, Evanston, IL 60208, USA \\ ${ }^{2}$ Sorbonne Universités, UPMC Univ Paris 06, UMR 7095, Institut d'Astrophysique de Paris, F-75014, Paris, France \\ ${ }^{3}$ CNRS, UMR 7095, Institut d'Astrophysique de Paris, F-75014, Paris, France \\ ${ }^{4}$ Laboratoire AIM-Paris-Saclay, CEA/DSM/IRFU, CNRS, Universite Paris Diderot, F-91191 Gif-sur-Yvette, France \\ ${ }^{5}$ Department of Physics and Astronomy, Johns Hopkins University, Baltimore, MD 21218, USA \\ Received 2015 June 5; accepted 2015 September 3; published 2015 October 8
}

\begin{abstract}
Studying how nuclear star clusters (NSCs) form and how they are related to the growth of the central massive black holes (MBHs) and their host galaxies is fundamental for our understanding of the evolution of galaxies and the processes that have shaped their central structures. We present the results of a semi-analytical galaxy formation model that follows the evolution of dark matter halos along merger trees, as well as that of the baryonic components. This model allows us to study the evolution of NSCs in a cosmological context, by taking into account the growth of NSCs due to both dynamical-friction-driven migration of stellar clusters and star formation triggered by infalling gas, while also accounting for dynamical heating from (binary) MBHs. We find that in situ star formation contributes a significant fraction (up to $\sim 80 \%$ ) of the total mass of NSCs in our model. Both NSC growth through in situ star formation and that through star cluster migration are found to generate NSC - host galaxy scaling correlations that are shallower than the same correlations for MBHs. We explore the role of galaxy mergers on the evolution of NSCs and show that observational data on NSC-host galaxy scaling relations provide evidence of partial erosion of NSCs by MBH binaries in luminous galaxies. We show that this observational feature is reproduced by our models, and we make predictions about the NSC and MBH occupation fraction in galaxies. We conclude by discussing several implications for theories of NSC formation.
\end{abstract}

Key words: galaxies: evolution - galaxies: formation - galaxies: nuclei - Galaxy: center quasars: supermassive black holes

\section{INTRODUCTION}

Over the past two decades, high-resolution observations with the Hubble Space Telescope have shown that massive stellar clusters reside at the photometric and dynamical centers of most intermediate- and low-luminosity galaxies of all Hubble types (Carollo et al. 1998; Matthews et al. 1999; Böker et al. 2002; Balcells et al. 2003; Graham \& Guzmán 2003; Côté et al. 2006). With sizes in the range $2-10 \mathrm{pc}$ and masses in the range $10^{5}-10^{8} M_{\odot}$, these nuclear clusters (NCs) have central densities up to $\sim 10^{6-7} M_{\odot} \mathrm{pc}^{-3}$, making them the densest stellar systems observed (e.g., Phillips et al. 1996; Walcher et al. 2005).

Nuclear star clusters (NSCs) are observed to be larger and brighter, and to follow different structural scaling relations than globular clusters. The NSC half-light radii scale with their total mass roughly as $r_{\mathrm{h}} \sim M_{\mathrm{NSC}}^{0.5}$, while globular clusters have $r_{\mathrm{h}} \approx 3 \mathrm{pc}$ irrespective of their luminosity, albeit with a large scatter (Harris 1996). However, the NSC mass distribution overlaps with that of globular clusters at its low-mass end, suggesting a possible connection between the two types of stellar clusters (e.g., Côté et al. 2006; Turner et al. 2012).

NSCs have been studied in detail for large samples of galaxies in different environments. Observational work has revealed that the nucleation fraction of galaxies is similar for galaxies belonging to very different environments, including the Virgo, Fornax, and Coma Clusters, as well as galaxies in the field (Côté et al. 2006; Turner et al. 2012; Baldassare et al. 2014; den Brok et al. 2014). On average, $80 \%$ of all galaxies of all Hubble types with magnitude in the range
$-20 \lesssim M_{B} \lesssim-12$ contain a well-defined central cluster. Hence, NSCs appear to be ubiquitous structures in galaxies, although they tend to be missing in galaxies brighter than magnitude $M_{B} \sim-20$ and fainter than $M_{B} \sim-12$ (e.g., van den Bergh 1986; Côté et al. 2006). The real nucleated fraction could be, however, larger than the value constrained from observations given that the morphological complexity and high surface brightness often characterizing the center of galaxies can make NSCs difficult to identify, especially in massive spheroids.

High-resolution spectroscopic surveys have been used to place constraints on NSC ages and star formation histories (Bender et al. 2005; Rossa et al. 2006; Walcher et al. 2006). The common finding emerging from these studies is that NSCs are characterized by a complex star formation history with a mixture of morphological components and different stellar populations spanning a wide range of characteristic ages from $10 \mathrm{Myr}$ to $10 \mathrm{Gyr}$. Cluster ages and masses are also found to depend on the host galaxy Hubble type, with NSCs in earlytype spirals being older and more massive than those of latetype spirals. The luminosity-weighted ages of NSCs are typically smaller than the ages of host galactic disks, indicating that the growth of the nuclei is a continuous and ongoing process occurring during and after most of the host galaxy was formed (Rossa et al. 2006).

Age estimates for most NSCs remain, however, susceptible to large uncertainties owing to the fact that the light is often dominated by young stellar populations while at the same time the mass is dominated by the old stars. The inability to infer spectroscopically the age of old cluster components can 
introduce a bias toward younger ages. The bulk of the stellar population is typically found in an older and spatially more extended stellar component (Carson et al. 2015). This appears to be the case for the Milky Way, in which $\gtrsim 80 \%$ of the stars inside the inner parsec formed more than $5 \mathrm{Gyr}$ ago, while luminous massive stars dominate the central cluster light within $\sim 0.5$ pc (Bender et al. 2005; Pfuhl et al. 2011; Lauer et al. 2012; Yusef-Zadeh et al. 2012).

NSC old and young stellar populations also differ morphologically. Seth et al. (2006) showed that the three edge-on late-type galaxies IC 5052, NGC 4206, and NGC 4244 have nuclei that are strongly flattened along the plane of their host galaxy disks. Such NSCs show evidence for young stellar components distributed onto a disk-like or ring structure superimposed on an older, more spherical component. In the edge-on spiral galaxy NGC 4244, old and young stellar components both show clear signs of rotation (Seth et al. 2008b). Seth et al. (2006) suggested that the presence of such multiple rotating morphological components and the presence of a flattened young cluster aligned with the major axis of the host galaxy point toward an in situ buildup of NCs, whereby stars form episodically in compact nuclear disks and then lose angular momentum or heat vertically to form an older spheroidal structure. It has been noted, however, that both rotation and the presence of young structural components do not exclude other formation mechanisms, such as episodic accretion of young star clusters in the central part of the galaxy due to dynamical friction (Antonini 2014). In the case of NGC 4244 , for example, it is likely that accreation of star clusters has contributed at least in part to the growth of its NSC (Hartmann et al. 2011; De Lorenzi et al. 2013).

Since the early studies, it has been realized that NSC masses correlate fairly well with galaxy properties such as bulge velocity dispersion and bulge and galaxy total luminosity (Balcells et al. 2003; Böker et al. 2004; Rossa et al. 2006). The existence of such correlations suggests that the formation of NSCs is intimately connected to the formation and evolution of the host galaxy (e.g., Leigh et al. 2015). Given that NSCs and massive black holes (MBHs) are found to coexist in some galaxies, that in these systems they have comparable masses, and that both follow tight correlations with galaxy properties, it is also natural to ask whether NSCs and MBHs are somehow connected to each other.

NSCs and MBHs are known to coexist in galaxies with masses $\sim 10^{10} M_{\odot}$ (González Delgado et al. 2008; Seth et al. 2008b); galaxies with masses lower than this value show clear evidence for nucleation but little evidence for an $\mathrm{MBH}$. Conversely, galaxies with masses above $\sim 10^{11} M_{\odot}$ are dominated by MBHs but show no evidence for nucleation (e.g., Wehner \& Harris 2006; Neumayer \& Walcher 2012). Ferrarese et al. (2006) found that the nuclei and MBHs obey a similar scaling relation linking their mass to the virial mass of the host galaxy. More recently, Graham (2012b) obtained NSC - host galaxy correlations using a sample in which he excluded what were likely to be nuclear stellar disks from the galaxy sample of Ferrarese et al., while including an additional 13 NSCs in galaxies with velocity dispersions out to about $200 \mathrm{~km} \mathrm{~s}^{-1}$. Graham found that NSC and MBH masses follow different scaling correlations with host galaxy properties. Other authors confirmed this result, showing that the mass of NSCs scales with the host galaxy spheroid's velocity dispersion as $M_{\mathrm{NSC}} \sim \sigma^{2}$, while the mass of MBHs follows the much steeper relation $M_{\mathrm{MBH}} \sim \sigma^{5}$ (Erwin \& Gadotti 2012; Graham 2012b; Leigh et al. 2012; Kormendy \& Ho 2013; Scott \& Graham 2013; den Brok et al. 2014). Although this might suggest that MBHs and NSCs did not form from the same mechanism (Antonini 2013), whether the formation of MBHs is connected to the evolution of NSCs and whether the two types of central objects grow together or in competition from the same physical process remain unclear.

The observational findings mentioned above provided motivation for theoretical work aimed at understanding how NSCs form and how their evolution is linked to the evolution of their host galaxy. Two models have been suggested for the formation of NSCs: (i) the cluster infall scenario, in which stellar clusters are driven to the galactic nucleus by dynamical friction, merge, and build up an NSC (Tremaine et al. 1975); (ii) the nuclear star formation scenario, in which gas falls into the nucleus and forms stars (e.g., Milosavljević 2004; McLaughlin et al. 2006). Owing to the inherent complexity of gas dynamics in star formation, which makes the second of these two processes difficult to model, theoretical work has been mostly directed toward the cluster infall scenario (but see Aharon \& Perets 2015). However, both dissipative and dissipationless processes are likely to play an important role in NSC formation (Antonini et al. 2015).

Theoretical studies have employed two different methodologies: $\mathrm{N}$-body simulations and semi-analytical modeling. $\mathrm{N}$ body techniques are used to simulate the last stage of inspiral and merger of stellar clusters in the inner region of galaxies. These models have shown that a star cluster merger scenario can explain without obvious difficulties the observed properties of NSCs, including their density and velocity dispersion profiles (e.g., Bekki et al. 2004; Capuzzo-Dolcetta \& Miocchi 2008; Antonini et al. 2012; Antonini 2014; Perets \& Mastrobuono-Battisti 2014). While these studies make reliable predictions about the aspect of NSCs, they suffer from the fact that the adopted initial conditions are often not well motivated, hence the need to rely on semi-analytical models. These are used to make predictions for the appearance of star clusters, of known mass and radius, in the center of galaxies, and to compute the accumulated mass at the center through dynamical friction migration over a Hubble time. In semi-analytical models, the rate at which the NSC grows by accreting young clusters is estimated from empirical cluster formation rates, dynamical friction timescales, and dissolution times (e.g., Agarwal \& Milosavljević 2011; Arca-Sedda \& CapuzzoDolcetta 2014; Gnedin et al. 2014). Studies based on semianalytical approaches have demonstrated that the NSC - host galaxy property scaling relations and their half-mass radiusmass relation are both consistent with formation by star cluster accretion (Antonini 2013).

All previous calculations assumed NSC formation to take place in isolated galactic spheroids, thus neglecting the role of galaxy evolution, mergers, and the role of in situ star formation. Also, these former idealized attempts could not explore the details of the interplay between MBH and NSC evolution. In this paper, we present a semi-analytical galaxy formation model $(\mathrm{GxeV})$ that allows us to shed light on exactly these points, i.e., it allows us to assess the role of galaxy mergers, $\mathrm{MBH}$ mergers, and nuclear star formation on the growth of NSCs. We follow the formation and evolution of galaxies, MBHs and NSCs along cosmic history, including the growth of NSCs due to both central migration of stellar clusters and 
in situ star formation, while also accounting for dynamical heating from (binary) MBHs.

The paper is organized as follows. In Section 2 we introduce and discuss the numerical methods employed in our study. Section 3 describes the sample data to which our numerical results are compared. In Sections 4 and 5 we describe the main results of our calculations and discuss some of their implications in Section 6. We summarize in Section 7.

\section{SEMI-ANALYTICAL MODELS}

We study the formation of NSCs and their coevolution with MBHs along the merger history of their host galaxies by semianalytical techniques.

First, we consider a purely dissipationless formation model in which stellar clusters form and migrate to the center of a galaxy through dynamical friction. We also consider a second semi-analytical model, which, unlike the first, follows the formation and merger history of galaxies (in both their dark matter and baryonic components). This allows us to study the formation, evolution, and disruption of NSCs in a selfconsistent way, accounting not only for the dynamical-frictiondriven inspiral of stellar clusters to the nucleus but also for star formation in the nuclear gas and for the effect of galaxy and MBH mergers.

We begin in this section by describing our methodology, highlighting in particular the simplifying approximations that are made in our models.

\subsection{Cluster Inspiral Model (CliN)}

In our semi-analytical model CliN a galactic nucleus forms through the orbital decay (via dynamical friction) and merger of star clusters in the central region of a galaxy. This code adopts a rather idealized model of an isolated galactic spheroid and follows the consecutive inspiral of stellar clusters along their orbits until they decay into the galactic center or they are disrupted in the process. This relatively simple approach has the advantage that the details of the tidal interaction of the clusters with the background galaxy and central $\mathrm{MBH}$ are easily included, and in a relatively precise manner. On the other hand, as previously stated, this model has the important limitation that galaxy evolution and dissipative processes that can lead to star formation episodes in the galactic center are neglected. Another basic assumption made in the CliN model is that MBHs are already in place before NSCs grow around them; moreover, we assume that all galaxies contain initially an $\mathrm{MBH}$.

The code is essentially the same as that used by Antonini (2013), and we direct the reader to that paper for a more detailed description. Briefly, we represent the galaxy spheroid by a simple power-law density model: $\rho(r)=\rho_{0}\left(r / r_{0}\right)^{-\gamma}$, where $\rho_{0}=(3-\gamma) M_{\mathrm{sph}} / 4 \pi r_{0}^{3}$. The latter expression assumes that the density of the galactic bulge follows a Dehnen (1993) profile, $r \sim r^{-4}$, at $r \gg r_{0}$. Given a mass for the central $\mathrm{MBH}$, $M_{\mathrm{MBH}}$, we compute the galaxy density profile slope, scale radius, spheroidal mass, and galaxy velocity dispersion by using the corresponding scaling relations that link these properties to $M_{\mathrm{MBH}}$ (Graham \& Driver 2007; Gültekin et al. 2009; Graham 2012a). The galaxy effective radius, $R_{\text {eff }}$, was derived from the size-mass relation given by Equation (32) of Shen et al. (2003). The ratio $R_{\text {eff }} / r_{0}$ follows from Equations (4) and (17) of Dehnen (1993), which give $R_{\text {eff }} / r_{0}=(1.8,1.5,1)$ for $\gamma=(1,1.5,2)$.

We assign the stellar cluster masses using the cluster initial mass function, $d n / d m_{\mathrm{gc}} \propto m_{\mathrm{gc}}^{-2}$ (Bik et al. 2003; de Grijs et al. 2003), and limiting mass values of $m_{\min }=10^{2} M_{\odot}$, $m_{\max }=10^{7} M_{\odot}$. The stellar clusters are assumed to form continuously over the age of the galaxy.

As commonly done, we assume that the clusters have initially the same distribution as the stars in the galaxy, and that initially a fixed fraction $f_{\mathrm{gc}}=0.05$ of stars form in strongly bound stellar clusters. The latter is approximately consistent with the typical cluster formation efficiency for the Milky Way found by Kruijssen (2012), and it is similar to the value adopted in previous studies (Gnedin et al. 2014). Gnedin et al. showed that by adopting a power-law mass function $d n / d m_{\mathrm{gc}} \propto m_{\mathrm{gc}}^{-2}$ and a fraction $f_{\mathrm{gc}}=0.04$ of clustered star formation, one can reproduce the observed density profile of clusters in M87.

We assume that the central properties of a stellar cluster remain unchanged during inspiral and that $r_{t}>r_{K}$, where $r_{K}$ is the cluster core radius and $r_{t}$ is the cluster tidal (limiting) radius given by (King 1962)

$$
r_{t}=\alpha \frac{\sigma_{K}}{\sqrt{2}}\left(\frac{3}{r} \frac{d \phi}{d r}-4 \pi G \rho\right)^{-1 / 2},
$$

with $\phi$ the sum of galactic, MBH, and NSC potentials, and $\alpha$ a "form factor" that depends on the density distribution within the cluster. The mass of a King model is then related to its tidal radius and velocity dispersion via the expression

$$
G m_{\mathrm{gc}} \approx \frac{\sigma_{K}^{2} r_{t}}{2}
$$

We note that although at first order the central properties of the clusters are not expected to change during inspiral, the stars in an NSC formed from cluster infalls should arguably end up with the same rms velocity as the galaxy host, which appears to be consistent with observations (see Leigh et al. 2015).

Given a cluster of central velocity dispersion $\sigma_{K}$, the time evolution of its orbital radius is

$$
r(t)=\left[r_{\text {in }}^{3-\gamma}-\frac{(3-\gamma)^{3}}{(4-\gamma) \sqrt{2 \gamma}} \frac{r_{0}^{-\gamma}}{4 \pi G \rho_{0}} F(\gamma) \ln \Lambda \sigma_{k}^{3} \times t\right]^{\frac{1}{3-\gamma}}
$$

where $r_{\text {in }}$ is the initial separation from the center, for the Coulomb logarithm we take $\ln \Lambda=6$, and $F(\gamma)$ is given in Equation (1) of Merritt et al. (2004). We approximately account for the dynamical dissolution of the clusters due to their collisional evolution and interaction with the external tidal field of the galaxy by only evolving clusters with dynamical friction time shorter than their dissolution time (Equation (11) below).

The cluster orbits are evolved using Equation (3) up to a maximum integration time of $10 \mathrm{Gyr}$, and the NSC mass is obtained by summing up all the mass transported by the infalling clusters within a radius $r_{\mathrm{NSC}}$. The NSC radius, $r_{\mathrm{NSC}}$, is computed from the recursive relation given by Equations (14)(16) of Antonini (2013). As we evolve the cluster orbits, we compute their tidal disruption radius due to the galaxy, $\mathrm{MBH}$, and preexisting NSC gravitational fields, adopting a cluster core radius $r_{K}=1 \mathrm{pc}$, roughly equal to the median value of the 
core radii listed in Harris's compilation of Galactic star clusters (Harris 1996). When the clusters reach their tidal disruption radius, the remaining core mass is dispersed around the radius of disruption. When evaluating the final NSC density distribution, we make the assumption that the stars from the disrupted clusters are isotropically distributed around the center. We note that in reality the clusters will distribute initially in a disk-like structure, but this will morph into a more spherical distribution over a fraction of the nuclear relaxation timescale (Antonini 2014).

An $\mathrm{MBH}$ binary formed during a galaxy merger leaves an imprint on the galactic nucleus in the form of a mass deficit, $M_{\mathrm{ej} \text {;in }}$, a decrease in the mass of the nucleus due to ejection of stars on intersecting orbits (Bekki \& Graham 2010). We account for this by subtracting a mass $M_{\mathrm{ej} \text {; bin }} \approx 0.5 \times$ $N_{\mathrm{m}} M_{\mathrm{MBH}}$ (Merritt 2006), where $N_{\mathrm{m}}$ is the predicted number of major mergers after the last major accretion event, from the final accumulated mass inside $r_{\text {NSC }}$. The number of mergers $N_{\mathrm{m}}$ is obtained from the galaxy mass using the distributions shown in Figure (2) of Haehnelt \& Kauffmann (2002), where in order to convert luminosity to mass we used a constant mass-to-light ratio $M / L_{V}=3 M_{\odot} / L_{\odot}$, as sometimes adopted in the literature for an old stellar population (e.g., Gnedin et al. 2014).

Finally, we compare the resulting NSC density profile, obtained after $10 \mathrm{Gyr}$ of evolution, to the density profile, $\rho(r)$, of the galaxy and define a galaxy as nucleated if at some radius the NSC stellar density was found to be higher than that of the background galaxy. The latter condition is based on the fact that if the NSC densities are below the galaxy density at all radii, then it would be difficult to observationally distinguish the two components.

\subsection{Galaxy Formation Model}

Our GxeV is based on that of Barausse (2012), which was further improved in Sesana et al. (2014). More precisely, Barausse (2012) presented a semi-analytical galaxy formation model tracking the evolution of baryonic structures along dark matter merger trees. These trees are produced with an extended Press-Schechter formalism, modified in order to reproduce the results of $N$-body simulations of dark matter halos (Parkinson et al. 2008), while the baryonic structures include the hot, largely unprocessed intergalactic medium, galactic disks and spheroids (in both their stellar and cold, chemically enriched interstellar medium components), a low angular momentum reservoir of cold nuclear gas available for accretion onto the central MBH (Granato et al. 2004; Lapi et al. 2014), and the MBH itself. These baryonic components are interconnected by a plethora of interactions, schematically summarized in Figure 1. Also included are environmental and tidal interactions between galaxies during mergers, following simple semianalytical recipes (Taffoni et al. 2003; Boylan-Kolchin et al. 2008). Moreover, Sesana et al. (2014) later improved this model by refining the star formation law-adding in particular an explicit dependence on the metallicity (Krumholz et al. 2009), as well as a distinction between pseudo-bulges forming from bar instabilities and classical bulges created by major mergers - and by devising more realistic prescriptions for the evolution of the spins of MBHs with cosmic time.

For this work, we have further ameliorated the model by including the formation and evolution of NSCs (see Figure 1). More precisely, we consider two possible formation channels for these objects, namely, one where NSCs form from the dynamical-friction-driven migration to the nuclear regions of star clusters created farther out in the galaxy, and one in which NSCs form in situ as a result of star formation in the nuclear regions. Note that we assume no high-redshift seeds for the NSCs, i.e., the NSC mass grows from zero at high redshifts to its present-day value through the two aforementioned channels (this growth being modulated/hindered by the effect of galaxy and black hole mergers; see Section 2.2.3).

In the $G x e V$ models described below we define as nucleated any galaxy containing a central cluster more massive than $10^{4}$ $M_{\odot}$, as this value corresponds approximately to the lower limit of the observed NSC mass distribution (e.g., den Brok et al. 2014). We also define an early-type (late-type) galaxy as one with bulge-to-total mass ratio $B / T>0.7(<0.7)$. For each of the GxeV models that we present, we simulate about 1300 galaxies with dark matter masses ranging from $10^{10}$ to $10^{15} M_{\odot}$.

\subsubsection{Migration Channel}

To implement the dynamical-friction-driven migration of star clusters to the central nucleus, we first assume that star clusters are created during star formation events with efficiency $f_{\mathrm{gc}}$, in both galactic bulges and disks. In bulges, Barausse (2012) and Sesana et al. (2014) assumed a volumetric star formation rate $\dot{\rho}_{\star}$, while in disks they considered a vertically averaged rate of star formation $\dot{\Sigma}_{\star}$. Therefore, we assume that star clusters form in bulges and disks, and that the total mass of the star cluster population (in both the bulge and disk), $M_{\mathrm{gc}}$, changes with rate

$$
\dot{M}_{\text {formation }}^{\mathrm{gc}}=f_{\mathrm{gc}} \times\left(4 \pi \int \dot{\rho}_{\star} r^{2} d r+2 \pi \int \dot{\Sigma}_{\star} r d r\right),
$$

where we choose $f_{\mathrm{gc}}=0.07$, which is the typical value in the Milky Way (Kruijssen 2012). We stress that plausible values $f_{\mathrm{gc}}=0.05-0.2$ would only mildly impact the normalization of our results for the $M_{\mathrm{NSC}}-\sigma$ relation and would not affect its slope significantly. In addition, we have tried a model with a variable $f_{\mathrm{gc}}$ set to $0.07,0.04$, and 0.5 in disk, quiescent, and starburst galaxies, respectively; these correspond approximately to the values of Figure 4 in Kruijssen (2012). The results of this model were found to not significantly differ from those of models with a fixed $f_{\mathrm{gc}}$. Thus, for the sake of simplicity, we present in what follows only the results of a model with fixed $f_{\mathrm{gc}}=0.07$.

We then assume that the star clusters formed in the bulge are spatially distributed in the same way as the bulge stellar population, i.e., if that population has density profile $\rho_{\text {bulge }}^{\star}(r)$, the volumetric number density $n_{\mathrm{gc}}^{\text {bulge }}$ of star clusters is assumed to satisfy

$$
p_{\mathrm{gc}}^{\mathrm{bulge}}(r) \equiv \frac{n_{\mathrm{gc}}^{\mathrm{bulge}}(r)}{N_{\mathrm{gc}}^{\mathrm{bulge}}}=\frac{\rho_{\mathrm{bulge}}^{\star}(r)}{M_{\mathrm{bulge}}^{\star}},
$$

where $N_{\mathrm{gc}}^{\text {bulge }}$ is the total number of star clusters in the bulge and $M_{\text {bulge }}^{\star}$ is the total bulge mass in stars. Note that following Barausse (2012), we assume a Hernquist distribution for $\rho_{\text {bulge }}^{\star}(r)$; we refer to Barausse (2012) for more details on the choice of the scaling radius for this density profile. 


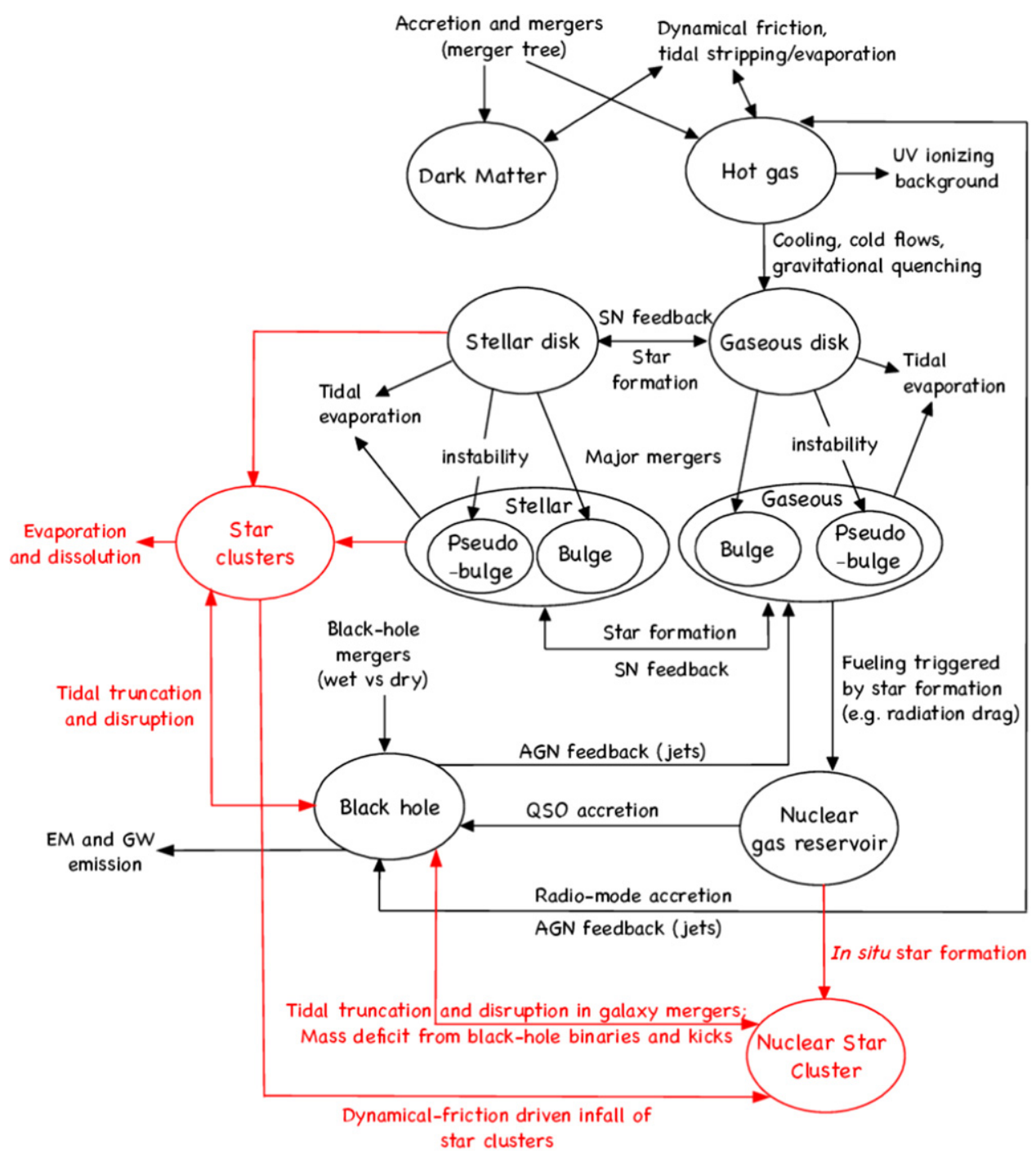

Figure 1. Schematic representation of the model of Barausse (2012; in black; including the improvements described in Sesana et al. 2014) with the additions described in this paper (in red) to model the formation and evolution of NSCs.

Similarly, we assume that the disk's star clusters are distributed according to the same surface density profile as the overall stellar content of the disk, i.e., the superficial number density $\nu_{\mathrm{gc}}^{\text {disk }}(r)$ will satisfy

$$
p_{\mathrm{gc}}^{\text {disk }}(r) \equiv \frac{\nu_{\mathrm{gc}}^{\text {disk }}(r)}{N_{\mathrm{gc}}^{\text {disk }}}=\frac{\sum_{\mathrm{disk}}^{\star}(r)}{M_{\mathrm{disk}}^{\star}},
$$

where again $N_{\mathrm{gc}}^{\text {disk }}$ is the total number of star clusters in the disk and $M_{\text {disk }}^{\star}$ is the mass of the stellar disk. Again, for $\Sigma_{\text {disk }}^{\star}(r)$ we follow Barausse (2012) and adopt an exponential profile (see Barausse 2012 for more details on the calculation of the scaling radius for this distribution).

As for the mass distribution of the newly formed star clusters, we assume a power-law mass function $d p_{\mathrm{gc}}^{\mathrm{X}} / d m_{\mathrm{gc}} \propto$ $m_{\mathrm{gc}}{ }^{-2}$ (with $X=$ bulge, disk). Combined with Equations (5) and (6), this gives the distribution functions for the bulge and disk star clusters

$$
\pi_{\mathrm{gc}}^{\text {bulge }} \equiv \frac{1}{N_{\mathrm{gc}}^{\text {bulge }}} \frac{d^{2} N_{\mathrm{gc}}^{\text {bulge }}}{d m_{\mathrm{gc}} d V}=\frac{d p_{\mathrm{gc}}^{\text {bulge }}}{d m_{\mathrm{gc}}}=A \frac{p_{\mathrm{gc}}^{\text {bulge }}(r)}{m_{\mathrm{gc}}^{2}}
$$

$$
\pi_{\mathrm{gc}}^{\mathrm{disk}} \equiv \frac{1}{N_{\mathrm{gc}}^{\text {disk }}} \frac{d^{2} N_{\mathrm{gc}}^{\text {disk }}}{d m_{\mathrm{gc}} d S}=\frac{d p_{\mathrm{gc}}^{\text {disk }}}{d m_{\mathrm{gc}}}=A \frac{p_{\mathrm{gc}}^{\mathrm{disk}}(r)}{m_{\mathrm{gc}}^{2}},
$$

where $d V$ and $d S$ are units of volume and surface. We assume that these distribution functions are valid for masses $m_{\mathrm{gc}}$ for individual star clusters between $m_{\min }=10^{2} M_{\odot} \quad$ and $m_{\max }=10^{6}-10^{7} M_{\odot}$, and the normalization factor

$$
A=\frac{m_{\max } m_{\min }}{m_{\max }-m_{\min }}
$$

ensures that the integral over all masses and over the whole volume (or surface) is 1 . Note that in our runs we assume $m_{\max }=10^{7} M_{\odot}$; using the dissipationless model described in Section 2.1, we find that reducing $m_{\max }$ to $10^{6} M_{\odot}$ has the effect of changing the normalization of the scaling relations by a factor of $\sim 10$, but did not change their overall slopes.

These distribution functions can then be used to calculate approximately how much mass is lost by the population of star clusters owing to their collisional evolution and interaction with the external tidal field of the galaxy ("dynamical 
dissolution"):

$$
\begin{aligned}
& \dot{M}_{\text {dissolution }}^{\mathrm{gc}} \\
& =\frac{M_{\mathrm{bulge}}^{\star}}{M_{\mathrm{bulge}}^{\star}+M_{\mathrm{disk}}^{\star}} M_{\mathrm{gc}} \int \frac{\pi^{\mathrm{bulge}}\left(r, m_{\mathrm{cl}}\right)}{t_{\mathrm{tid}}\left(r, m_{\mathrm{cl}}\right)} 4 \pi r^{2} d r d m_{\mathrm{cl}} \\
& +\frac{M_{\mathrm{disk}}^{\star}}{M_{\mathrm{bulge}}^{\star}+M_{\mathrm{disk}}^{\star}} M_{\mathrm{gc}} \int \frac{\pi^{\mathrm{disk}}\left(r, m_{\mathrm{cl}}\right)}{t_{\mathrm{tid}}\left(r, m_{\mathrm{cl}}\right)} 2 \pi r d r d m_{\mathrm{cl}},
\end{aligned}
$$

with

$$
t_{\text {tid }} \approx 10 \mathrm{Gyr}\left(\frac{m_{\mathrm{cl}}}{2 \times 10^{5} M_{\odot}}\right)^{\alpha} P(r)
$$

and

$$
P(r)=41.4\left(\frac{r}{\mathrm{kpc}}\right)\left(\frac{V_{\mathrm{vir}}}{\mathrm{km} \mathrm{s}^{-1}}\right)^{-1},
$$

$V_{\text {vir }}$ being the halo's virial velocity (e.g., Gnedin et al. 2014). Recent $N$-body simulations show that $\alpha \approx 2 / 3$ (Gieles \& Baumgardt 2008), which we adopt here. Also, note that for simplicity we do not track separately the mass in star clusters in the bulge and that in the disk, but simply follow the evolution of the total mass in star clusters $M_{\mathrm{gc}}$ (see Equation (4)). In Equation (10) (and in Equations (13) and (16) below) we therefore simply assume that the mass of star clusters in the disk is $\approx\left[M_{\text {disk }}^{\star} /\left(M_{\text {bulge }}^{\star}+M_{\text {disk }}^{\star}\right)\right] M_{\mathrm{gc}}$, and that the mass of star clusters in the bulge is $\approx\left[M_{\text {bulge }}^{\star} /\left(M_{\text {bulge }}^{\star}+M_{\text {disk }}^{\star}\right)\right] M_{\mathrm{gc}}$.

In a similar fashion, we account for the evaporation of star clusters in isolation through

$$
\begin{aligned}
& \dot{M}_{\text {evaporation }}^{\mathrm{gc}} \\
& =\frac{M_{\mathrm{bulge}}^{\star}}{M_{\mathrm{bulge}}^{\star}+M_{\mathrm{disk}}^{\star}} M_{\mathrm{gc}} \int \frac{\pi^{\text {bulge }}\left(r, m_{\mathrm{cl}}\right)}{t_{\mathrm{ev}}\left(m_{\mathrm{cl}}\right)} 4 \pi r^{2} d r d m_{\mathrm{cl}} \\
& +\frac{M_{\mathrm{disk}}^{\star}}{M_{\mathrm{bulge}}^{\star}+M_{\mathrm{disk}}^{\star}} M_{\mathrm{gc}} \int \frac{\pi^{\mathrm{disk}}\left(r, m_{\mathrm{cl}}\right)}{t_{\mathrm{ev}}\left(m_{\mathrm{cl}}\right)} 2 \pi r d r d m_{\mathrm{cl}},
\end{aligned}
$$

where

$$
t_{\mathrm{ev}}=\frac{17 m_{\mathrm{cl}}}{2 \times 10^{5} M_{\odot}} \mathrm{Gyr} .
$$

Note that because the evaporation timescale does not depend on the star cluster's location (since it describes evaporation in isolation), Equation (13) can be simplified to

$$
\dot{M}_{\text {evaporation }}^{\mathrm{gc}}=A M_{\mathrm{gc}} \int \frac{m_{\mathrm{gc}}^{-2}}{t_{\mathrm{ev}}\left(m_{\mathrm{cl}}\right)} d m_{\mathrm{cl}} .
$$

Note that our models do not include the possibility of close encounters with giant molecular clouds, which in some cases could significantly reduce the clusters' lifetime. Also, they rely on necessarily simplified models for the galaxy. A more realistic model for the galactic potential might somewhat change the timescales introduced above. However, we note that analytical models of NSC formation indicate that the final NSC mass is not much affected by the assumed slope of the galactic background density profile (see Section 4 of Antonini 2013).

The total mass in star clusters also decreases because the individual star clusters fall toward the central NSC, under the effect of dynamical friction, i.e.,

$$
\begin{aligned}
& \dot{M}_{\text {infall }}^{\mathrm{gc}} \\
& =\frac{M_{\mathrm{bulge}}^{\star}}{M_{\text {bulge }}^{\star}+M_{\mathrm{disk}}^{\star}} M_{\mathrm{gc}} \int \frac{\pi^{\text {bulge }}\left(r, m_{\mathrm{cl}}\right)}{t_{\mathrm{df}, \text { bulge }}\left(r, m_{\mathrm{cl}}\right)} 4 \pi r^{2} d r d m_{\mathrm{cl}} \\
& +\frac{M_{\mathrm{disk}}^{\star}}{M_{\text {bulge }}^{\star}+M_{\mathrm{disk}}^{\star}} M_{\mathrm{gc}} \int \frac{\pi^{\text {disk }}\left(r, m_{\mathrm{cl}}\right)}{t_{\mathrm{df}, \mathrm{disk}}\left(r, m_{\mathrm{cl}}\right)} 2 \pi r d r d m_{\mathrm{cl}} .
\end{aligned}
$$

The dynamical friction timescale in the bulge is dominated by the interaction with the stellar background, and we therefore follow Binney \& Tremaine (2008) (Equation (8.12)):

$$
\begin{aligned}
t_{\mathrm{df}, \text { bulge }}\left(r, m_{\mathrm{cl}}\right) \approx & 15 \mathrm{Gyr} \frac{6}{\log \Lambda}\left(\frac{r}{5 \mathrm{kpc}}\right)^{2} \\
& \times \frac{\sigma}{100 \mathrm{~km} \mathrm{~s}^{-1}} \frac{10^{7} M_{\odot}}{m_{\mathrm{cl}}},
\end{aligned}
$$

where $\sigma$ is the bulge velocity dispersion (related to the halo's virial velocity by $\sigma \approx 0.65 V_{\text {vir }}$; Ferrarese 2002) and we choose $\log \Lambda=6$. As for the dynamical friction in the disk, we account for the interaction with both the stellar and gas component, i.e.,

$$
\begin{gathered}
t_{\mathrm{df}, \mathrm{disk}}\left(r, m_{\mathrm{cl}}\right)^{-1}=t_{\mathrm{df}, \mathrm{disk}}^{\star}\left(r, m_{\mathrm{cl}}\right)^{-1}+t_{\mathrm{df}, \mathrm{disk}}^{\mathrm{gas}}\left(r, m_{\mathrm{cl}}\right)^{-1} \\
t_{\mathrm{df}, \mathrm{disk}}^{\star}=\frac{L}{\dot{L}_{\star}}, \quad t_{\mathrm{df}, \mathrm{disk}}^{\mathrm{gas}}=\frac{L}{\dot{L}_{\mathrm{gas}}}, \quad L \approx m_{\mathrm{cl}} V_{\mathrm{vir}} r, \\
\dot{L}_{\star}=\left(\frac{G m_{\mathrm{cl}}}{\sigma_{\star}}\right)^{2} \Sigma_{\star}, \quad \dot{L}_{\mathrm{gas}}=\left(\frac{G m_{\mathrm{cl}}}{\sigma_{\mathrm{gas}}}\right)^{2} \Sigma_{\mathrm{gas}},
\end{gathered}
$$

where $L$ is the orbital angular moment of a star cluster of mass $m_{\mathrm{cl}}$ belonging to the disk (and thus moving with velocity $\sim V_{\text {vir }}$ at a separation $r$ from the galactic center), while the angular momentum loss due to dynamical friction is expressed (Agarwal \& Milosavljević 2011) in terms of the surface densities $\Sigma_{\star}$ and $\Sigma_{\text {gas }}$ of the disk's stellar and gaseous components, the velocity dispersion of the stars in the disk $\sigma_{\star}$, and the velocity dispersion/sound speed of the gas in the disk $\sigma_{\text {gas }} \approx 0.1 \sigma_{\star}$ (Dutton \& van den Bosch 2009). Note that for $\sigma_{\star}$ we follow Kregel et al. (2005) and assume $\sigma_{\star} \approx 0.29 V_{\max }$, where $V_{\max }$ is the maximum rotational velocity inside the disk. Also observe that in Equation (18) we are summing the inverses of the timescales, so that the infall rates due to the dynamical friction from the gas and that due to the stars get summed in Equation (16).

Not all of the mass of the star clusters falling toward the NSC eventually accretes onto it, because star clusters get truncated owing to the galactic tidal field and tidally disrupted by the central $\mathrm{MBH}$. To account for these effects, we assume that the rate of change of the NSC mass due to infall of star clusters is

$$
\dot{M}_{\text {infall }}^{\mathrm{NSC}}=\dot{M}_{\text {infall }}^{\mathrm{gc}} \mathcal{F},
$$

where clearly the major difficulty lies in computing the factor $0 \leqslant \mathcal{F} \leqslant 1$. Since it would be computationally prohibitive to evolve the infall of the individual star clusters self-consistently within our semi-analytical galaxy formation model, we calculate $\mathcal{F}$ with the "monolithic" CliN model of Section 2.1, in order to derive an easy-to-use analytical expression. 
More specifically, approximating a star cluster as a King model, its tidal radius at a distance $r_{\mathrm{NSC}}$ from the center of a galaxy containing an $\mathrm{MBH}$ and an NSC at its center is

$$
\begin{aligned}
r_{t} \approx & \frac{\sigma_{K}}{\sqrt{2}}\left[4 \pi G \rho_{0}\left(\frac{r_{\mathrm{NSC}}}{r_{0}}\right)^{-\gamma} \frac{\gamma}{3-\gamma}\right. \\
& \left.+\frac{3 G\left(M_{\mathrm{CMO}}\right)}{r_{\mathrm{NSC}}^{3}}\right]^{-1 / 2},
\end{aligned}
$$

where $M_{\mathrm{CMO}}=M_{\mathrm{MBH}}+M_{\mathrm{NSC}}$ is the total mass of the central massive objects (i.e., MBH plus NSC mass), and for the galaxy density profile we adopted the power-law model $\rho(r)=\rho_{0}\left(r / r_{0}\right)^{-\gamma}$. Note that to be consistent with the Hernquist profile used in $G x e V$, we set $\gamma=1$ in Equation (22). Also, the distance $r_{\mathrm{NSC}}$ is to be set to the outer radius of the NSC, i.e., the distance from the galaxy center below which the star cluster is assumed to have become part of the NSC. We assume $r_{\mathrm{NSC}}=5 r_{\mathrm{h}}$, where $r_{\mathrm{h}}$ is the NSC half-mass radius. Our detailed prescription for $r_{\mathrm{h}}$ will be presented in Equation (25) below. For a King model, the truncated mass $m_{\mathrm{gc}}\left(\sigma_{K}\right)$ of the star cluster is then related to its tidal radius via Equation (2).

Using the CliN model described in Section 2.1, we find that more than $90 \%$ of the NSC mass comes from star clusters with initial mass $\gtrsim 0.1 m_{\max }$, and that the (mass-weighted) mean central velocity dispersion of the star clusters contributing to the nucleus growth is $\left\langle\sigma_{K}\right\rangle \approx 20 \mathrm{~km} \mathrm{~s}^{-1}$. When setting $m_{\max }=10^{7} M_{\odot}$, the average initial mass of these star clusters is $\left\langle m_{\mathrm{gc}, \text { in }}\right\rangle \approx 2.5 \times 10^{6} M_{\odot}$. Assuming that all star clusters that decay to the center have similar central properties $\left(\sigma_{K}\right)$, and that these properties do not change during the infall, we have

$$
\mathcal{F} \approx \frac{m_{\mathrm{gc}}\left(\left\langle\sigma_{K}\right\rangle\right)}{\left\langle m_{\mathrm{gc}, \text { in }}\right\rangle},
$$

where $m_{\mathrm{gc}}\left(\left\langle\sigma_{K}\right\rangle\right)$ is given by Equations (2) and (22). Finally, to account for the possibility that a star cluster may undergo complete tidal disruption before it decays to a radius $r_{\mathrm{NSC}}$, we compare the tidal radius given by Equation (22) with the core radius $r_{K} \approx 1 \mathrm{pc}$ of the star cluster. If $r_{t}<r_{K}$, we set $\mathcal{F}=0$.

\subsubsection{Star Formation Channel}

As mentioned above, NSCs may also form by star formation in the cold gas accumulating in the galactic center. In the galaxy formation model of Barausse (2012) (and in the improved version of this model used by Sesana et al. 2014), transfer of cold gas to a low angular momentum nuclear reservoir-available for accretion onto the central $\mathrm{MBH}$ - is assumed to be correlated with star formation events in the galactic bulge through the relation (Granato et al. 2004; Haiman et al. 2004; Lapi et al. 2014)

$$
\dot{M}_{\text {infall }}^{\text {res }}=A_{\text {res }} \psi_{\mathrm{b}},
$$

where $\psi_{\mathrm{b}}$ is the star formation rate in the gaseous bulge and $A_{\text {res }} \sim 10^{-2}-10^{-3}$ is a free parameter, which we set to $A_{\text {res }} \approx 6 \times 10^{-3}$ in this paper (as in Sesana et al. 2014). In the model of Barausse (2012) and Sesana et al. (2014), star formation in the bulge is associated with major galactic mergers and (less importantly) with bar instabilities in the galactic disk.

Note that the physical mechanism responsible for the loss of angular momentum of the cold gas and its transfer to this nuclear reservoir may be the radiation drag caused by the newly formed stars (Umemura 2001; Kawakatu \& Umemura 2002; Kawakatu et al. 2003), or the reshuffling/shocks of the gas as a consequence of disk instabilities or mergers. More generally, a correlation between bulge star formation and $\mathrm{MBH}$ accretion/ growth is expected based on the $M-\sigma$ relation for MBHs and on the parallelism between the quasar luminosity and star formation history (Granato et al. 2004; Haiman et al. 2004; Lapi et al. 2014).

The reservoir's gas is then made available to accrete onto the $\mathrm{MBH}$ on the viscous timescale. Whenever the resulting viscous accretion rate exceeds $A_{\text {Edd }} \dot{M}_{\text {Edd }}\left(\dot{M}_{\text {Edd }}\right.$ being the Eddington mass accretion rate and $A_{\mathrm{Edd}}$ a free parameter), we truncate the accretion rate to that value. To allow for moderately superEddington accretion in the case in which MBHs form from light Population III star seeds (of mass $\sim 200 M_{\odot}$ at $z \sim 15-20$ ) (Madau \& Rees 2001), we set $A_{\text {Edd }}=2.2$ for that seed model. This is because some amount of super-Eddington accretion is known to be needed to reconcile light $\mathrm{MBH}$ seeds with the quasar luminosity function at high redshift (Madau et al. 2014). We also consider two "heavy"-seed models (namely, that of Volonteri et al. 2008 and that of Koushiappas et al. 2004) whereby MBHs grow from seeds of $\sim 10^{5} M_{\odot}$ at $z \sim 15-20$, in which case we set $A_{\text {Edd }}=1$.

On top of these prescriptions, in this paper we assume that the gas in the nuclear reservoir also forms stars, which are assumed to contribute to the NSC. To compute the star formation rate, we need to choose a size for the reservoir. It is natural to assume that this size is comparable to the observed size of NSCs. More specifically, we assume that the reservoir is disk-like and has a (vertically averaged) exponential density profile, whose scale radius we choose such that the reservoir has the same half-mass radius $r_{\mathrm{h}}$ as the NSC. The latter is assumed to be

$$
r_{\mathrm{h}}=3 \operatorname{pc} \max \left(\sqrt{\frac{M_{\mathrm{dyn}}}{10^{6} M_{\odot}}}, 1\right),
$$

with $M_{\mathrm{dyn}}=M_{\mathrm{res}}+M_{\mathrm{NSC}}$ the dynamical mass of the nucleus (including the gas in the nuclear region). Note that this scaling is inspired by observations of the size of NSCs, which is found to scale with the square root of the luminosity (Turner et al. 2012).

Once a density profile for the reservoir is specified, we apply a star formation law similar to that used by Sesana et al. (2014) for galactic disks. Note that Sesana et al. (2014) improved on Barausse (2012) by considering different modes of star formation in classical bulges forming from major mergerswhere star formation is assumed to take place in "bursts" of duration comparable to the bulge dynamical time-as opposed to disks and pseudo-bulges arising from bar instabilities of disks - where star formation is assumed to happen in a "quiescent" mode, described by the prescription of Krumholz et al. (2009). Since star formation in the central region of our Galaxy is known to be weaker by at least a factor of 10 relative to what would be expected based on the observed gas densities (Kruijssen et al. 2014), it seems more appropriate to use the 
"quiescent" star formation mode for our nuclear reservoir. ${ }^{6}$ Following therefore Sesana et al. (2014) and Krumholz et al. (2009), we assume that the star formation in the reservoir takes place on a timescale $t_{\mathrm{SF}}$ and involves only a fraction $f_{\mathrm{c}}$ of the reservoir's gas, thus allowing us to write an approximate star formation rate in the reservoir as

$$
\dot{M}_{\mathrm{res}}^{\mathrm{sf}}=\frac{f_{\mathrm{c}} M_{\mathrm{res}}}{t_{\mathrm{SF}}} .
$$

Note that this simple prescription does not account for the spatial distribution of the star formation activity, e.g., the observational fact that younger stellar components seem to be more centrally concentrated than the rest of the NSC (see, e.g., Georgiev \& Böker 2014).

The fraction $f_{\mathrm{c}}$ of cold gas available for star formation is set by the fraction of molecular gas for metallicities $Z^{\prime}>0.01$ (in solar units), when star formation happens in molecular clouds. At lower metallicities $Z^{\prime}<0.01$, star formation takes place in the atomic phase (Krumholz 2012). In general, $f_{\mathrm{c}}$ is expected to decrease as the metallicity decreases, but recent observations of nearby spirals and dwarfs (Bigiel et al. 2010), as well as the Small Magellanic Cloud (Bolatto et al. 2011), show that it levels off at 2\% (Genzel et al. 2015).

We can thus summarize the dependence of $f_{\mathrm{c}}$ on the metallicity by the explicit expression (Krumholz et al. 2009; Sesana et al. 2014)

$$
f_{\mathrm{c}}= \begin{cases}1-\left[1+\left(\frac{3}{4} \frac{s}{1+\delta}\right)^{-5}\right]^{-1 / 5}, & \text { if } f_{\mathrm{c}}>2 \%, \\ 2 \%, & \text { otherwise }\end{cases}
$$

with

$$
\begin{gathered}
s=\ln (1+0.6 \chi) /\left(0.04 \Sigma_{1} Z^{\prime}\right), \\
\chi=0.77\left(1+3.1 Z^{\prime 0.365}\right), \\
\delta=0.0712\left(0.1 s^{-1}+0.675\right)^{-2.8}, \\
\Sigma_{1}=\Sigma_{\mathrm{res}} /\left(M_{\odot} \mathrm{pc}^{-2}\right) .
\end{gathered}
$$

The timescale $t_{\mathrm{SF}}$ is instead given by (Krumholz et al. 2009; Sesana et al. 2014)

$$
t_{\mathrm{SF}}^{-1}=(2.6 \mathrm{Gyr})^{-1} \times \begin{cases}\left(\frac{\Sigma_{\text {res }}}{\Sigma_{\text {th }}}\right)^{-0.33}, & \Sigma_{\text {res }}<\Sigma_{\text {th }}, \\ \left(\frac{\Sigma_{\text {res }}}{\Sigma_{\text {th }}}\right)^{0.34}, & \Sigma_{\text {res }}>\Sigma_{\text {th }}\end{cases}
$$

with $\Sigma_{\text {th }}=85 M_{\odot} \mathrm{pc}^{-2}$. This expression is obtained by assuming that star formation happens in clouds, and the two branches appear according to whether the cloud density is determined by internal processes or by the external pressure (in galaxies with sufficiently high surface densities). Also, note that the typical cloud mass does appear in this expression because it is related to the gas surface density by identifying it with the local Jeans mass and by assuming marginal

\footnotetext{
6 Observe that although star formation takes place quiescently in our model's nuclear reservoir, we also assume (see Equation (24)) that the reservoir's feeding is triggered by star formation events in the bulge, i.e., mainly by turbulence-driven bursts of star formation following major mergers.
}

gravitational stability of the reservoir. For the surface density of the reservoir, in both Equations (27) and (28), we choose $\Sigma_{\text {res }} \approx M_{\text {res }} /\left(2 \pi r_{\text {res }}^{2}\right), \quad r_{\text {res }}$ being the scale radius of the reservoir's exponential surface density profile. This is the central value of the density for an exponential surface density profile. While this choice is quite arbitrary, we note that at high surface densities the combination $f_{\mathrm{c}} / t_{\mathrm{SF}}$ has a weak dependence on $\Sigma_{\text {res }}$, namely, $f_{\mathrm{c}} / t_{\mathrm{SF}} \propto \Sigma_{\text {res }}^{0.34}$. Therefore, a different choice of $\Sigma_{\text {res }}$ by a factor of 2 (5) only changes $\dot{M}_{\text {res }}^{\text {sf }}$ as given by Equation (26) by $0.1(0.24)$ dex.

We stress that Equation (26) is admittedly a rough prescription for the star formation in the nuclear regions. However, it seems to work reasonably well when compared to observations of the star formation rates in the central $500 \mathrm{pc}$ of the Milky Way (the "central molecular zone"). Indeed, in Figure 2 we compare the predictions of our star formation prescription (where in Equations (27) and (28) we identify $\Sigma_{\text {res }}$ with the surface gas density represented in the horizontal axis) with measurements of the star formation in the central molecular zone at different angular scales (and thus different average gas surface densities).

In light of this reasonable agreement, we chose not to include any feedback effects (AGNs or supernovae) when considering star formation in the nuclear regions. We stress, however, that both kinds of feedback are included when considering the bulge and disk star formation (see Figure 1, and Barausse 2012). They therefore indirectly affect the star formation in the nuclear region, since the feeding of the reservoir depends on the larger-scale galactic evolution via Equation (24).

\subsubsection{Effect of Galaxy Mergers and Black Hole Binaries}

Delays between galaxy and black hole mergers.-When two dark matter halos merge, the two galaxies, being more compact than the halos, initially retain their identity and are slowly brought together by dynamical friction. During this evolution, environmental effects such as tidal stripping and tidal evaporation become important and contribute to remove mass from the smaller galaxy. ${ }^{7}$ When the galaxies finally merge, within a few dynamical friction timescales after the halo merger, the MBHs of the two galaxies are expected to form a binary system.

This binary is slowly brought together by a variety of effects, i.e., dynamical friction at large separations, and at smaller $\lesssim$ pc separations by gas interactions (if sufficient gas is present in the nuclear regions) or by interaction with stars if the NSC has a sufficiently dense core, or by triaxiality of the central potential. Gas interactions (and in particular planet-like migration) might bring an $\mathrm{MBH}$ binary to merger on timescales $\lesssim 10^{7}$ $10^{8} \mathrm{yr}$ (Haiman et al. 2009; Colpi 2014), while three-body interactions with stars might require timescales up to $\sim \mathrm{Gyr}(\mathrm{Yu}$ 2002) or more. In fact, it is not completely obvious that an $\mathrm{MBH}$ binary would merge at all (within a Hubble time) in the presence of three-body interactions with stars alone. This is known as the "final-parsec" problem (Begelman et al. 1980), but recent numerical simulations suggest that in triaxial merger remnants (such as those that would be expected from a recent galaxy merger) $\mathrm{MBH}$ binaries might coalesce on timescales of a few Gyr (Khan et al. 2011; Vasiliev 2014; Vasiliev et al. 2014, 2015). Rotation in flattened galaxy models has

\footnotetext{
7 We refer to Barausse (2012) for more details about how we compute the dynamical-friction timescale and these environmental effects.
} 


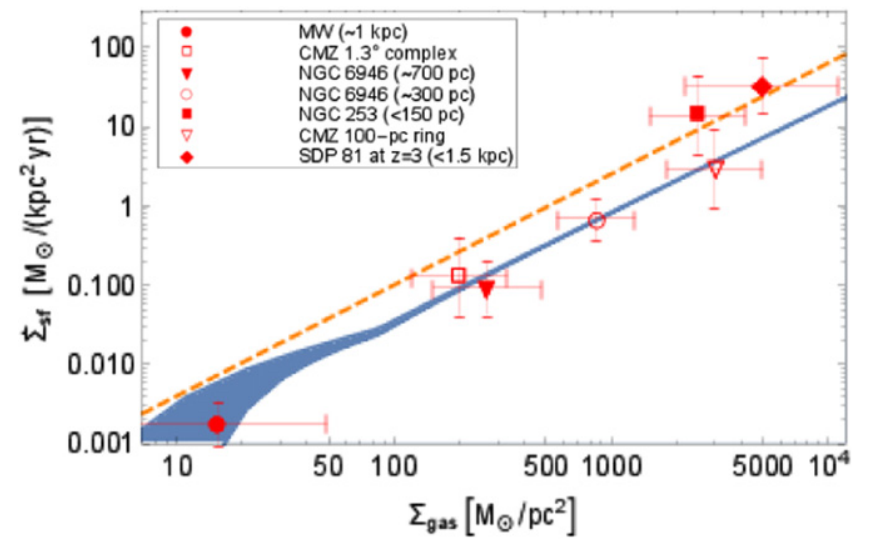

Figure 2. Comparison between our star formation prescription in the nuclear region-denoted by a blue line, the width of the line representing the scatter of the predicted star formation rate with metallicity, which we assume in this figure to vary between 1 and 4 solar units - and the measured star formation in the Milky Way's central molecular zone (CMZ) as reported by Kruijssen et al. (2014); the average star formation rate in the central $150 \mathrm{pc}$ of NGC 253, as reported in Table 6 of Leroy et al. (2015); the Milky Way's star formation rate at galactocentric distance of $\sim 1 \mathrm{kpc}$ and that of NGC 6946 at galactocentric distances of $\sim 300$ and $\sim 700$ pc, as reported by Kennicutt \& Evans (2012) in their Figure 7; and the measured nuclear star formation in the star-forming galaxy SDP 81 at $z \approx 3$ (Dye et al. 2015; Swinbank et al. 2015). For comparison, we also show by a dashed orange line the prediction of the Kennicutt-Schmidt law (Kennicutt 1998).

also been suggested to help drive $\mathrm{MBH}$ binaries to coalescence (see Holley-Bockelmann \& Khan 2015). Also, if an MBH binary stalls at $\sim$ pc separation, a later galaxy merger may add another $\mathrm{MBH}$ to the system, forming an $\mathrm{MBH}$ triple. At least for large $\sim 10^{8} M_{\odot} \mathrm{MBH}$ masses, triple $\mathrm{MBH}$ interactions may trigger binary mergers (and possibly ejection of the smallest $\mathrm{MBH}$ ) on timescales $\lesssim 10^{8} \mathrm{yr}$ (Hoffman \& Loeb 2007). Because of the uncertainty about the mechanisms described above, we adopt here a set of minimal simplified prescriptions to estimate the "delay" between galaxy and $\mathrm{MBH}$ mergers. These prescriptions are presented in the following.

In gas-rich environments, defined by the criterion $M_{\text {res }}>M_{\mathrm{MBH}, 1}+M_{\mathrm{MBH}, 2}$, we assume that the delay is simply given by the viscous time of the nuclear gas. At a distance $r$ from the central MBH, the viscous time is simply $t_{\nu} \sim r^{2} / \nu, \nu$ being the kinematic viscosity. The latter can be approximately expressed in terms of the gas velocity $v$ and the Reynolds number $\mathcal{R}$ as $\nu \sim r v / \mathcal{R}$ (Duschl et al. 2000; Frank et al. 2002, p. 398). Since the kinematic viscosity is huge for accretion disks, it is natural to assume that $\mathcal{R}$ is the critical Reynolds number $\mathcal{R}_{c} \sim 10^{2}-10^{3}$ marking the onset of turbulence. Indeed, laboratory experiments find that once this critical Reynolds number is reached, the viscosity increases significantly (Duschl et al. 2000; Frank et al. 2002, p. 398). Assuming now that $r \sim G\left(M_{\mathrm{MBH}, 1}+M_{\mathrm{MBH}, 2}\right) / \sigma^{2}$ is the binary's influence radius and that $v \sim \sigma$, we can write the "delay" in gas-rich nuclear environments as (Granato et al. 2004)

$$
t_{\text {delay,gas }} \sim t_{\nu} \sim \mathcal{R}_{c} t_{\text {dyn }},
$$

where $t_{\mathrm{dyn}}=G\left(M_{\mathrm{MBH}, 1}+M_{\mathrm{MBH}, 2}\right) / \sigma^{3}$ is the dynamical time at the influence radius. In this paper we set $\mathcal{R}_{c}=10^{3}$. This prescription does indeed yield delays $\lesssim 10^{7}-10^{8} \mathrm{yr}$. in gas-rich environments, as expected.

In gas-poor enviroments (i.e., $M_{\mathrm{res}}<M_{\mathrm{MBH}, 1}+M_{\mathrm{MBH}, 2}$ ), three-body interactions with stars dominate and bring the
MBHs together on a timescale (Begelman et al. 1980)

$$
\begin{aligned}
& t_{\text {delay }, \text { stars }} \sim 5 \operatorname{Gyr}\left(\frac{a_{\mathrm{gr}}}{4.5 \times 10^{-2} \mathrm{pc}}\right)^{4} \\
& \times\left[\frac{q}{(1+q)^{2}}\left(\frac{M_{\mathrm{MBH}, 1}+M_{\mathrm{MBH}, 2}}{10^{8}}\right)^{3}\right]^{-1},
\end{aligned}
$$

where $q=M_{\mathrm{MBH}, 2} / M_{\mathrm{MBH}, 1} \leqslant 1$, and $a_{\mathrm{gr}}$ is the radius at which gravitational-wave emission becomes dominant over threebody interactions with stars at driving the binary's evolution, i.e.,

$$
\begin{aligned}
a_{\mathrm{gr}} \approx & 1.65 \times 10^{-2} \mathrm{pc} \\
& \times\left[\frac{q}{(1+q)^{2}}\left(\frac{M_{\mathrm{MBH}, 1}+M_{\mathrm{MBH}, 2}}{10^{8} M_{\odot}}\right)^{3}\right. \\
& \left.\times\left(\frac{\sigma}{200 \mathrm{~km} \mathrm{~s}^{-1}}\right)\left(\frac{\rho_{\star}}{10^{3} M_{\odot} \mathrm{pc}^{-3}}\right)^{-1}\right]^{1 / 5} .
\end{aligned}
$$

Here the density $\rho_{\star}$ of the stellar background in which the binary moves is given by the maximum of the stellar bulge density and the density of the NSC. For the latter, we use the average NSC density within its half-mass radius, i.e., $\rho_{\mathrm{NSC}} \approx\left[M_{\mathrm{NSC}}-2\left(M_{\mathrm{MBH}, 1}+M_{\mathrm{MBH}, 2}\right)\right] /\left[(8 / 3) \pi r_{\mathrm{h}}^{3}\right], \quad$ where we decrease the mass of the NSC by twice the mass of the binary to approximately account for the mass deficit it causes on the NSC during its inspiral (see Equation (34) below). As for the stellar bulge density, our model assumes a Hernquist profile, as mentioned earlier. Comparisons to $\mathrm{N}$-body simulations (Sesana \& Khan 2015) show that the appropriate radius where this density profile needs to be evaluated in order to estimate $a_{\mathrm{gr}}$ correctly is the binary's mass influence radius, i.e., the radius at which the enclosed bulge mass (in stars) equals twice the binary mass.

In order to model triple $\mathrm{MBH}$ systems when they form, we utilize the results of Hoffman \& Loeb (2007). By using numerical simulations, Hoffman \& Loeb (2007) found that $\mathrm{MBH}$ triples typically trigger the merger of the two more massive components on timescales of $10^{8} \mathrm{yr}$ (on average) for binary masses of $\sim 6 \times 10^{8} M_{\odot}$ (and roughly comparable masses for the two components), while the lightest MBH may be ejected from the galaxy or be left wandering far from the galaxy's center, or less likely come back and coalesce with the remnant of the inner binary's merger. Moreover, the timescales for the merger of the inner binary present a lognormal scatter of about $1.4 \mathrm{dex}$ around the median value of $10^{8} \mathrm{yr}$, as shown in Figure 8 (upper panel) in Hoffman \& Loeb (2007). To extend these results to arbitrary masses, we rescale these timescales with the system's dynamical time at the binary's hardening radius, and we thus obtain an average "delay" due to $\mathrm{MBH}$ triple interactions given by

$$
t_{\text {delay,triple }} \sim 10^{8} \text { yr. }\left(\frac{M_{\mathrm{MBH}, 1}+M_{\mathrm{MBH}, 2}}{6 \times 10^{8} M_{\odot}}\right)^{1 / 4} \times \frac{8 q^{3 / 2}}{(1+q)^{3}} .
$$

Whenever a triple forms, we then extract the delay with which the two more massive objects merge from a lognormal distribution centered on this timescale, and with rms of 
1.4 dex. For simplicity, we also assume that the lightest MBH is ejected from the galaxy and therefore lost to the subsequent galaxy evolution.

Finally, Hoffman \& Loeb (2007) noted that triple MBH interactions are less effective at driving $\mathrm{MBHs}$ to merger in low-mass systems, where the third incoming MBH is ejected from the galaxy before it can shrink the inner binary to separations at which gravitational-wave emission is important. Indeed, the escape velocity from a galaxy scales as the velocity dispersion, i.e., $v_{\mathrm{esc}} \sim \sigma \sim M^{1 / 4}$, where we defined the mass of the inner binary, $M \equiv M_{\mathrm{MBH}, 1}+M_{\mathrm{MBH}, 2}$, and we used the Faber-Jackson relation $M \sim \sigma^{4}$. The separation $a_{\mathrm{gw}}$ at which an MBH binary is driven to coalescence by gravitational-wave emission within a Hubble time $t_{\mathrm{H}}$ can be obtained from Equation (30) (with the replacements $a_{\mathrm{gr}} \rightarrow a_{\mathrm{gw}}$ and $t_{\text {delay,stars }} \rightarrow t_{\mathrm{H}}$ ) and scales as $a_{\mathrm{gw}} \sim M^{3 / 4} q_{\nu}^{1 / 4}$ (with $q_{\nu}=$ $M_{\mathrm{MBH}, 1} M_{\mathrm{MBH}, 2} /\left(M_{\mathrm{MBH}, 1}+M_{\mathrm{MBH}, 2}\right)^{2}$ the symmetric mass ratio). In order for the third MBH to be able to shrink the inner binary to the separation $a_{\mathrm{gw}}$, the velocity $v_{3}$ of the third MBH should be lower than $v_{\mathrm{esc}}$ when the binary's separation is $a_{\mathrm{gw}}$. The energy of the third MBH can be estimated simply by energy equipartition as $E_{3} \sim M_{\mathrm{MBH}, 3} v_{3}^{2} \sim G M_{\mathrm{MBH}, 1} M_{\mathrm{MBH}, 2} / a_{\mathrm{gw}}$, from which one gets $v_{3} \sim M^{1 / 8} q_{\nu}^{3 / 8} q_{3}^{-1 / 2}$, with $q_{3}=M_{\mathrm{MBH}, 3} / M$. This, in turn, gives $v_{3} / v_{\mathrm{esc}} \sim M^{-1 / 8} q_{\nu}^{3 / 8} q_{3}^{-1 / 2}$. The normalization of this ratio can be estimated using the results of Hoffman \& Loeb (2007), who find $v_{\text {esc }} \sim 1400 \mathrm{~km} \mathrm{~s}^{-1}$ and $v_{3} \sim 750$ $\mathrm{km} \mathrm{s}^{-1}$ for $M \sim 6 \times 10^{8} M_{\odot}, q_{\nu} \sim 1 / 4$ and $q_{3} \sim 0.25-0.3$ (see their Figures 5 and 11), thus giving

$$
\frac{v_{3}}{v_{\mathrm{esc}}} \sim 0.5 \times \frac{q_{\nu}^{3 / 8}}{q_{3}^{1 / 2}\left[M /\left(6 \times 10^{8}\right)\right]^{1 / 8}} .
$$

Therefore, whenever this equation gives $v_{3}>v_{\text {esc }}$, we assume that the third $\mathrm{MBH}$ is ejected without driving the merger of the inner binary. This implies that triple MBH interactions become ineffective at driving the merger of binary systems with total mass $M \lesssim 2 \times 10^{6} \times\left(q_{\nu}^{3} / q_{3}^{4}\right) M_{\odot}$.

Mass deficit caused by black hole binaries.-The presence of an MBH binary has profound effects on the NSC, causing a "mass deficit" in the central regions of the galaxy. Indeed, during the binary's inspiral, three-body interactions with the stars in the nuclear region result in an exchange of energy between the binary (which becomes more and more bound) and the stars, to which large velocities are imparted that are capable of ejecting them from the galactic nucleus. Also, when the $\mathrm{MBH}$ binary finally merges, the resulting $\mathrm{MBH}$ remnant acquires a kick velocity up to a few thousand kilometers per second owing to the anisotropic emission of gravitational waves (Campanelli et al. 2007), and this also contributes to removing stars from the galactic core. A similar mass deficit may be caused by the ejection of the lightest MBH in a triple system.

We estimate the mass-ejection rate from the NSC due to the MBH binary's inspiral as

$$
\dot{M}_{\mathrm{ej}}^{\text {insp }} \approx\left(M_{\mathrm{MBH}, 1}+M_{\mathrm{MBH}, 2}\right) \frac{0.7 q^{0.2}+0.5 \ln \left(a_{\mathrm{h}} / a_{\mathrm{gr}}\right)}{t_{\text {delay, stars }}},
$$

where $q=M_{\mathrm{MBH}, 2} / M_{\mathrm{MBH}, 1} \leqslant 1$ is the binary's mass ratio and $a_{\mathrm{h}}$ is the semimajor axis at which the binary becomes "hard" (i.e., tightly bound).

The first term in the numerator of Equation (34) represents the mass scoured by the MBH binary before it becomes hard, where we have identified the ejected mass with the mass deficit as defined in Merritt (2006); the second term represents instead the mass ejected from $a_{\mathrm{h}}$ to $a_{\mathrm{gr}}$ (Merritt 2013, p. 544); the explicit expression for $a_{\mathrm{h}}$ is given, for instance, in Merritt (2013, p. 544), i.e.,

$$
a_{\mathrm{h}} \approx 0.27(1+q)^{-1}\left(\frac{M_{\mathrm{MBH}, 2}}{10^{7} M_{\odot}}\right)\left(\frac{\sigma}{200 \mathrm{~km} \mathrm{~s}^{-1}}\right)^{-2} \mathrm{pc},
$$

while for $a_{\mathrm{gr}}$ we utilize Equation (31). Also, in the denominator of Equation (34), we note the presence of the timescale $t_{\text {delay, }}$, stars, computed via Equations (30) and (31). That timescale accounts for the fact that both terms in the numerator where computed for $\mathrm{MBH}$ binaries in gas-poor environments; hence, the mass deficit at the numerator should be "spread" over the timescale characterizing stellar interactions. Of course, if the MBH binary lives in a gas-poor enviroment, in our model the inspiral lasts exactly $t_{\text {delay,stars, and the final }}$ mass deficit caused by the binary is $\sim\left(M_{\mathrm{MBH}, 1}+M_{\mathrm{MBH}, 2}\right)$ $\left[0.7 q^{0.2}+0.5 \ln \left(a_{\mathrm{h}} / a_{\mathrm{gr}}\right)\right]$. If the binary evolution is instead driven by gas interactions or by the formation of an $\mathrm{MBH}$ triple, that mass deficit is suppressed by a factor of $t_{\text {delay,gas }} / t_{\text {delay,stars }}$ or $t_{\text {delay,triple }} / t_{\text {delay, stars }}$, respectively. ${ }^{8}$

In addition to the mass deficit caused during the inspiral, when the MBH binary finally merges, the resulting $\mathrm{MBH}$ remnant acquires a kick, which, as mentioned above, can further remove mass from the NSC. We estimate this mass deficit as (Gualandris \& Merritt 2008)

$$
M_{\mathrm{ej}}^{\text {kick }} \approx 5\left(M_{\mathrm{MBH}, 1}+M_{\mathrm{MBH}, 2}\right)\left(V_{\text {kick }} / V_{\mathrm{esc}}\right)^{1.75},
$$

where $V_{\text {kick }}$ is the recoil velocity of the MBH remnant and $V_{\text {esc }}$ is the escape velocity from the galactic center. The latter can be easily calculated within our model, from the mass and density profiles of the bulge and NSC. As for $V_{\text {kick }}$, we follow Barausse (2012) and use the analytical formula of van Meter et al. (2010), which fits the results of numerical relativity simulations.

Similarly, in the case of triple MBH systems, if the lightest MBH gets ejected from the system before it can cause the inner binary to merge (i.e., in our model, if the ratio $v_{3} / v_{\text {esc }}$ given by Equation (33) is larger than 1), we assume that the ejected MBH causes a mass deficit $M_{\mathrm{ej}}^{\text {kick,triple }} \sim 5 M_{\mathrm{MBH}, 3}$, with $M_{\mathrm{MBH}, 3}$ the ejected MBH's mass. We stress that for simplicity we neglect the mass deficit caused by the recoil of the lightest $\mathrm{MBH}$ in the cases in which the triple interactions trigger the merger of the inner binary (i.e., in our model, when $\left.v_{3} / v_{\text {esc }}<1\right)$. Summarizing, the total mass deficit due to MBH coalescences is $M_{\mathrm{ej}}=M_{\mathrm{ej}}^{\mathrm{insp}}+M_{\mathrm{ej}}^{\mathrm{kick}}+M_{\mathrm{ej}}^{\text {kick,triple }}$.

\footnotetext{
Note that we have also tried setting the delays between galaxy and $\mathrm{MBH}$ mergers to very small values $t_{\text {delay }} \sim 10^{6}$ yr. irrespective of the mechanism driving the binary's evolution prior to the gravitational-wave-dominated phase. In this case, the mass deficit is always $\sim\left(M_{\mathrm{MBH}, 1}+M_{\mathrm{MBH}, 2}\right)$ $\left[0.7 q^{0.2}+0.5 \ln \left(a_{\mathrm{h}} / a_{\mathrm{gr}}\right)\right]$. This test confirms that our results for the NSC evolution are reasonably robust, and that the overall conclusions of this paper do not depend on our particular model for the delays.
} 
In addition to the effect of $\mathrm{MBH}$ binaries, we also account for the possible tidal disruption of NSCs by MBHs during galaxy mergers. Indeed, if a merger takes place between a galaxy ("1") that contains an NSC (but not an MBH) and another galaxy ("2") hosting an MBH (and possibly an NSC), the NSC of "galaxy 1" will be dragged by dynamical friction toward the $\mathrm{MBH}$ (and NSC if present) of galaxy 2 and will therefore be tidally truncated/disrupted, as in the case of star clusters falling toward the nucleus of an isolated galaxy. To model this effect, we assume that the NSC resulting from such a galaxy merger has mass

$$
M_{\mathrm{NSC}}=M_{\mathrm{NSC}, 1} \times \mathcal{F}+M_{\mathrm{NSC}, 2} .
$$

The fraction $0 \leqslant \mathcal{F} \leqslant 1$ accounts for the tidal truncation/ disruption effects and is calculated via Equations (2), (22), and (23), with $\sigma_{K}$ taken to be the velocity dispersion of "NSC 1" (which one can compute in terms of its mass and size). Note that calculating $\mathcal{F}$ in the same way as for star clusters is justified, at least to first approximation. Indeed, as mentioned earlier, the star clusters that contribute the most to Equation (23) have average initial mass $\left\langle m_{\mathrm{gc} \text {, in }}\right\rangle \approx 2.5 \times 10^{6} M_{\odot}$, which is of the same order of magnitude as a typical NSC mass. As in the case of star clusters, if $r_{t}<r_{K}=r_{\mathrm{h}}^{\mathrm{NSC} 1} / c$ (with $c=30$ a typical NSC concentration parameter), we assume that "NSC 1 " is fully disrupted and set $\mathcal{F}=0$. On the other hand, if both "galaxy 1" and "galaxy 2" have MBHs, we assume that the gravitational field of each massive $\mathrm{BH}$ protects its NSC from tidal truncation and disruption, and we set $\mathcal{F}=1$. Similarly, if neither galaxy contains an $\mathrm{MBH}$, we set $\mathcal{F}=1$.

\section{DATA SAMPLE}

We compare our models to observational data in order to gain insights on the reliability of such models. We then use our models to put constraints on the origin and evolution of NSCs and to understand how their properties are linked to the evolution of MBHs and their host galaxies.

Our sample of NSC objects was obtained by combining data from Scott \& Graham (2013), Erwin \& Gadotti (2012), and Neumayer \& Walcher (2012) and by estimating NSC object masses of the galaxies in the Fornax Cluster catalog of Turner et al. (2012).

Erwin \& Gadotti (2012) give mass estimates for a total of 18 galaxies that cover Hubble types from $\mathrm{S} 0$ to $\mathrm{Sm}$ with dynamically determined NSC masses and 15 Sa and later-type galaxies from Rossa et al. (2006) with NSC mass estimates based on high-resolution spectroscopy. Velocity dispersions for nine of these galaxies were obtained from Table 2 of Neumayer \& Walcher (2012), the rest from the HyperLeda database when available. Scott \& Graham (2013) constructed their sample of NSCs using photometric data from Ferrarese et al. (2006), Balcells et al. (2007), Graham \& Spitler (2009), and Graham (2012a). Table 1 of Scott \& Graham (2013) reports 76 galaxies containing an NSC with a well-determined mass, and total mass and velocity dispersion estimates for the majority of these. The uncertainties on the nuclear object masses are given by Ferrarese et al. (2006), Balcells et al. (2007), and Graham \& Spitler (2009) as 45\%, 33\%, and a factor of two, respectively.

We additionally obtained estimates for the stellar masses of the full galaxy, $M_{\mathrm{gx}}$, and the NSC, using the photometric data for the 43 early-type galaxies in the Fornax Cluster catalog of Turner et al. (2012). To determine $M_{\mathrm{gx}}$, we multiplied the total galaxy luminosity in the $K$ band given in the HyperLeda database by a mass-to-light ratio of $M / L_{K}=0.8$ (Bell \& de Jong 2001), based on the typical colors of the bulge population. Galaxy total $B$ magnitudes, $M_{B}$, and velocity dispersions, $\sigma$, were also taken from the HyperLeda database. The NSC masses were obtained by multiplying the published luminosities by the appropriate $M / L$, obtained by using the empirical correlations between color and $M / L$ given in Bell et al. (2003). Typical errors on $M_{\mathrm{NSC}}$ are $\sim 35 \%$.

After eliminating duplicate galaxies contained in more than one of the samples-for each galaxy we included in the collective sample the mass estimate with the smallest uncertainty-we collected a total of 89 galaxies with reliable estimates for both $M_{\mathrm{NSC}}$ and $\sigma, 127$ objects with determined $M_{\mathrm{NSC}}$ and $M_{\mathrm{gx}}$, and 208 galaxies with measured NSC mass and host galaxy total $B$ magnitude.

Although we were not able to obtain all galaxy properties for every object from the literature, our data collectively represent the largest sample to date of NSC and host galaxy physical properties. In Figure 3 we plot the NSC masses as a function of the velocity dispersion of the galaxy spheroid, as well as the galaxy mass, for early-type (filled circles) and late-type (open circles) systems.

\subsection{Data Analysis}

In order to investigate the correlation between NSCs and their host galaxy properties, we use a locally weighted linear regression model (Loess) to fit nonparametric curves to the data (Cleveland 1979; Cleveland \& Devlin 1988). Loess does not require one to specify a global function of any form to fit a model to the data, as it combines multiple low-order polynomial regression models in a $k$-nearest-neighbor-based metamodel. Unlike a more "standard" simple linear regression analysis, no assumption is made that the data can be fitted by a straight line. Loess scatter-plot smoothing can therefore be used to reveal complex relationships that could be overlooked with traditional parametric estimation strategies. The obvious tradeoff is that in general it is not possible to express a Loess model with a simple mathematical formula.

The smoothness of the Loess regression function is determined by the amount of data points used for the local fits, a quantity controlled by the span parameter, $\alpha$. Here we select an optimal value of $\alpha$ by using the generalized crossvalidation criterion (Craven \& Wahba 1979). The basic idea is that the optimal representation of the data is obtained by adopting the smoothing parameter that minimizes the meansquared error of the fit (Golub et al. 1979; Li 1985).

The solid thick black curves in Figure 3 are Loess interpolations obtained with the optimal smoothing parameters $\alpha=0.63$ and 0.71 for $M_{\mathrm{NSC}}$ versus $\sigma$ and $M_{\mathrm{gx}}$, respectively. The $95 \%$ variance bands of the Loess curves are shown as thin black curves.

The first noteworthy point is that the Loess curves shown in Figure 3 are characterized by a significant bending at $\sigma \gtrsim 100 \mathrm{~km} \mathrm{~s}^{-1}$ and $M_{\mathrm{gx}} \gtrsim 10^{10.5} M_{\odot}$, indicating NSC-host galaxy scaling relations that are flat or even declining for the most luminous galaxies. Moreover, the reconstructed variance bands follow the general trend of the Loess curves, indicating that it is unlikely that the nonlinear features present in the curves are due to random fluctuations in the data alone.

We tested whether or not a linear parametrization of the relations provides an adequate description of the data by using 

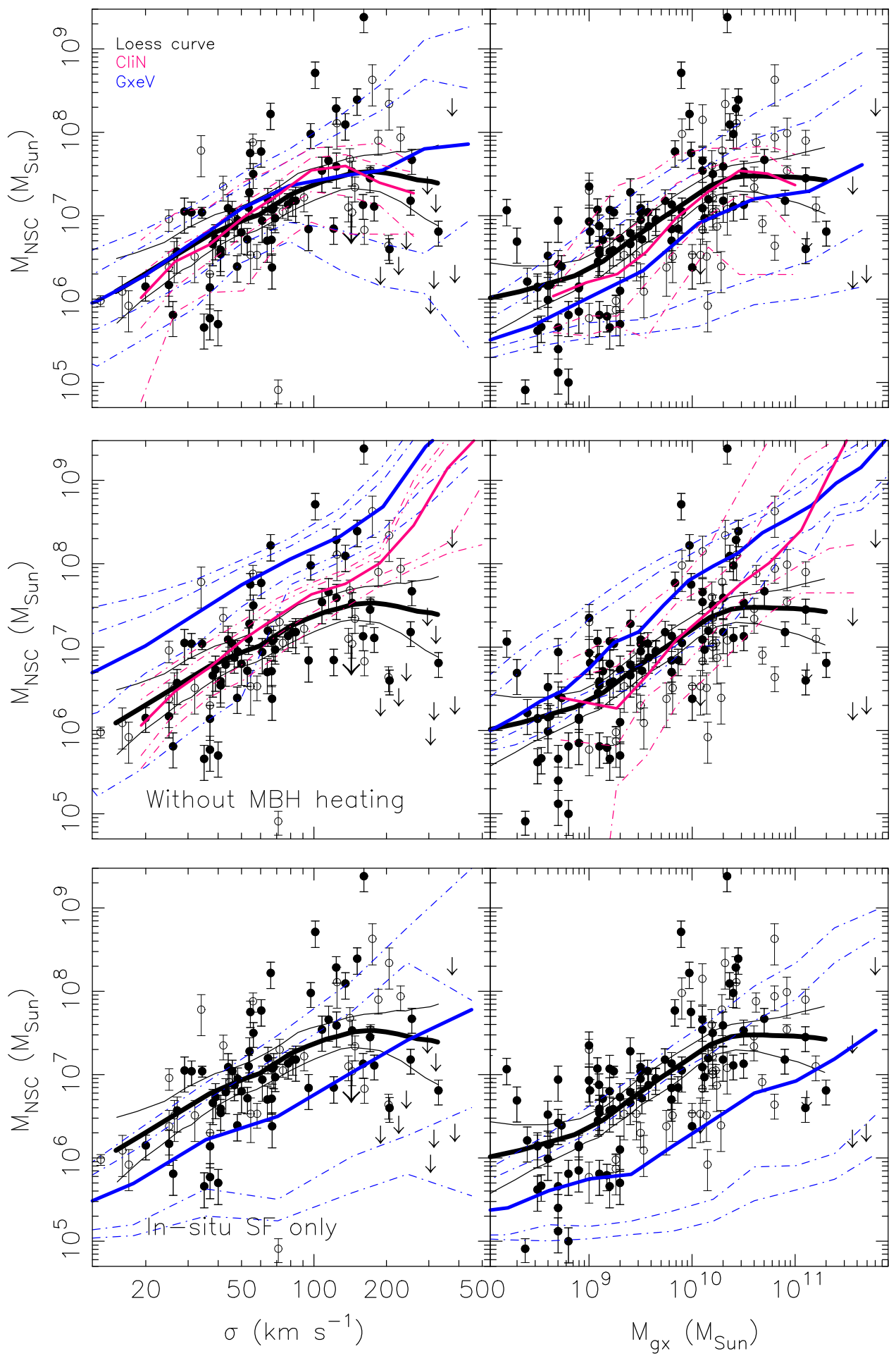

Figure 3. Top panels: $M_{\text {NSC }}$ against galaxy velocity dispersion (left panel) and galaxy total mass (right panel). Filled circles represent early-type galaxies (S0 or earlier), while open circles are late-type systems. Short (long) arrow symbols are NSCs in early-type (late-type) galaxies with only an upper limit on their mass (Neumayer \& Walcher 2012). Since it is likely that these systems do not contain an NSC, we decided not to include these upper limits in our regression analysis. Black lines are the computed Loess curves, and thin black lines give the corresponding 95\% variance bands. Red lines give the median of the CliN model, where only cluster inspirals are considered, and corresponding $70 \%$ and $90 \%$ confidence-level regions. Blue lines are the results of our semi-analytical model $\mathrm{GxeV}$ including cluster inspirals, in situ star formation, and processes related to hierarchical galaxy and MBH evolution. Dashed lines indicate the $70 \%$ and $90 \%$ confidence-level regions. Middle panels: same as top panels, but with MBH dynamical effects not included in the semi-analytical computations (see Section 4.1 for details). Bottom panels: same as top panels, but NSCs are allowed to grow only via in situ star formation (see Section 4.2 for details).

an $F$-test to compare the Loess fits to simpler weighted linear fits (Fox 1999). The $F$-tests showed that the null hypothesisi.e., that the bivariate Loess model yields no improvement in the fit over the linear regression-can be rejected at a high level of significance. For all three relations investigated here, the Loess curves provide a better description to the data than linear 


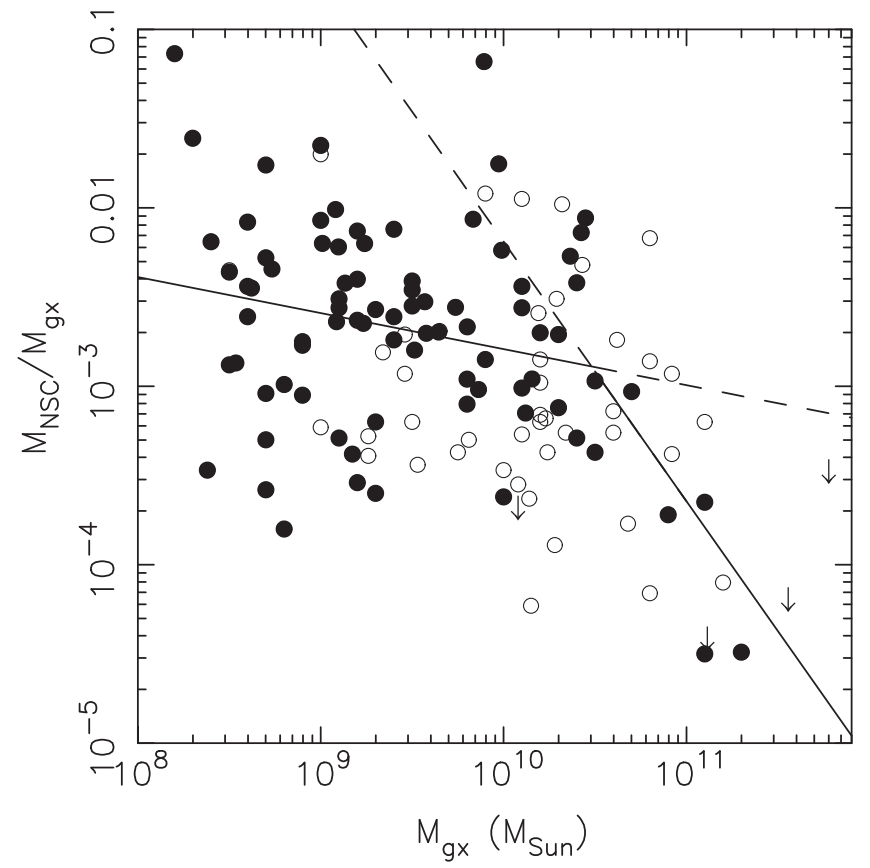

Figure 4. NSC mass fraction as a function of the total stellar mass of the host galaxy. Filled circles are early-type galaxies, open circles are late-type galaxies. Black lines give simple linear fits to the data with $M_{\mathrm{gx}} \lesssim 10^{10.5} M_{\odot}$ and with $M_{\mathrm{gx}} \gtrsim 10^{10.5} M_{\odot}$. In the low-mass regime the NSC-to-galaxy mass ratio shows a weak correlation with $M_{\mathrm{gx}}$, while in the more massive systems $\left(M_{\mathrm{gx}} \gtrsim 10^{10.5} M_{\odot}\right)$ there is a clear trend with $M_{\mathrm{gx}}$, with the NSC mass fraction decreasing rapidly with the galaxy mass. This is consistent with the flattening of the scaling relations found at high galaxy masses in Figure 3.

models at $>99 \%$ confidence level. This confirms that the nonlinear features present in the functional dependence of $M_{\mathrm{NSC}}$ on the galaxy properties are likely not due to noise variability in the data.

A complementary view is provided in Figure 4, where we plot the NSC mass fraction as a function of the total stellar mass of the host galaxy, i.e., $M_{\mathrm{NSC}} / M_{\mathrm{gx}}$ versus $M_{\mathrm{gx}}$. Evidently, in the low-mass regime $\left(M_{\mathrm{gx}} \lesssim 10^{10.5} M_{\odot}\right)$ the NSC -to-galaxy mass ratio shows a weaker correlation with total galaxy mass (with Kendall's rank correlation coefficient $\tau_{B}=-0.28$ and associated probability value $p=0.06$ ), while in the more massive systems $\left(M_{\mathrm{gx}} \gtrsim 10^{10.5} M_{\odot}\right)$ there is a clear and steeper trend with $M_{\mathrm{gx}}$, in the sense that the NSC mass fraction decreases rapidly with the galaxy mass. This is in contrast to the results of Scott \& Graham (2013), who claimed quite a steep decrease of the NSC mass ratio based on fits to the entire sample distribution.

We finally note that although our analysis demonstrates that current data are consistent with a significant bending of the NSC-host galaxy scaling relations, further observations and analysis, combined with theory, will be needed in order to refine our results. On the other hand, the broadening of the NSC mass distribution for the highest-luminosity galaxies, as well as the presence of low-mass nuclei $\left(\sim 10^{6} M_{\odot}\right)$ in highmass galaxies $\left(M_{\mathrm{gx}} \gtrsim 10^{11} M_{\odot}\right)$, certainly poses a serious concern for previous claims of the existence of fundamental correlations between NSC and host galaxy properties. Moreover, as we show in the following, the particular form of these relations is in agreement with what is expected on the basis of our semi-analytical models of NSC formation.

\section{RESULTS}

The red and blue lines in Figure 3 show the resulting NSC mass as a function of $\sigma$ obtained with CliN after $10 \mathrm{Gyr}$ of evolution and with $G x e V$ at $z=0$, respectively. These curves give the median output at a given $\sigma$ or $M_{\mathrm{gx}}$, as well as its $70 \%$ and $90 \%$ confidence-level regions, i.e., the regions containing, respectively, $70 \%$ and $90 \%$ of the NSCs produced by our models at a given stellar mass. The CliN model median can be fit by $M_{\mathrm{NSC}} \approx 10^{7} M_{\odot}\left(\sigma / 50 \mathrm{~km} \mathrm{~s}^{-1}\right)^{2}$ and $M_{\mathrm{NSC}} \approx 10^{7}$ $M_{\odot}\left(M_{\mathrm{gx}} / 10^{10} M_{\odot}\right)^{0.9}$ at $\sigma \lesssim 100 \mathrm{~km} \mathrm{~s}^{-1}$ and $M_{\mathrm{gx}} \lesssim 10^{10.5} M_{\odot}$, respectively. This appears to be in good agreement with analytical expectations-Antonini (2013) finds $M_{\mathrm{NSC}} \approx$ $10^{7} M_{\odot}\left(\sigma / 50 \mathrm{~km} \mathrm{~s}^{-1}\right)^{3 / 2}$, adopting an idealized isothermal sphere galaxy model. Similar scalings and a similar scatter around the median values are found with the galaxy evolution model $G x e V$, which takes into account both cluster inspirals and in situ star formation. The similarity between the results of the two models with and without in situ star formation suggests that dynamical friction migration of star clusters is a fundamental process for the growth of the nuclei. However, as we show below in Section 4.2, dissipative processes also play an important role, contributing a significant fraction of the total NSC masses in our models.

Figure 3 shows that at $\sigma \gtrsim 100 \mathrm{~km} \mathrm{~s}^{-1}$ and $M_{\mathrm{gx}} \gtrsim 10^{10.5} M_{\odot}$, the scaling relations produced with $C l i N$ (i.e., a purely dissipationless formation model) appear to flatten toward the most luminous galaxies, in agreement with the results of the data analysis of Section 3. However, a more careful analysis of the plot also shows that such a model fails to explain the existence of NSCs of mass $\gtrsim 10^{8} M_{\odot}$, which are clearly present in the observational data. The reason for this discrepancy is that in $C l i N$ we have assumed that all galaxies contain an $\mathrm{MBH}$, and that these $\mathrm{MBHs}$ are in place at the center of all initial galaxy models before the NSCs grow around them. These assumptions are not fully supported by our galaxy formation model (see Section 5) and artificially lead to an enhanced mass removal from the stellar clusters accreting onto the central NSC, because of the tidal field of the MBH.

The galaxy evolution model GxeV produces scaling relations at $z=0$ that are in remarkably good agreement with the observed correlations. The scatter around the median value clearly increases at the high-mass end of the NSC distribution. A large population of NSCs are produced at $\sigma \gtrsim 100 \mathrm{~km} \mathrm{~s}^{-1}$ that are significantly underweight with respect to what the same model would predict by simply extrapolating the scaling correlations and scatter from low $\left(M_{\mathrm{gx}} \lesssim 10^{10.5} M_{\odot}\right)$ to high galaxy masses. In the following section we argue that the formation of such "underweight" NSCs and, consequently, the apparent bending of the scaling correlations can be explained in terms of the interaction of the NSCs with their host galaxy MBHs.

\subsection{The Role of $\mathrm{MBHs}$}

In our semi-analytical models MBHs affect the formation and evolution of NSCs in two important ways: (i) The strong tidal field of the central MBH accelerates the mass loss from stellar clusters as they enter its sphere of influence and eventually disrupts them as they come closer than a distance 
$r_{\text {disr }}$, where

$$
\frac{M_{\mathrm{MBH}}}{\frac{4}{3} \pi r_{\mathrm{disr}}^{3}} \approx \rho(0) \approx \frac{9}{4 \pi G} \frac{\sigma_{K}^{2}}{r_{K}^{2}}
$$

After a cluster is disrupted, most of its stars are dispersed around $r_{\text {disr }}$, which will limit the nuclear density within this radius. Thus, a preexisting $\mathrm{MBH}$ will limit the amount of mass that can be transported to the center by decaying stellar clusters (Antonini 2013). As mentioned earlier (see Equation (37)), a similar effect applies to an NSC falling toward a central $\mathrm{MBH}$ after a galaxy merger. (ii) During galaxy mergers, $\mathrm{MBH}$ binaries form and harden by ejecting surrounding stars (Milosavljević \& Merritt 2001). By dynamically heating the NSC, an MBH binary will lower the NSC central stellar density, or fully destroy the NSC when the binary's mass is significantly larger than the NSC mass (Bekki \& Graham 2010). The kick imparted to the merger remnant by asymmetric emission of gravitational-wave radiation will remove additional mass from the galaxy nucleus (Gualandris \& Merritt 2008).

To demonstrate the role of MBHs in the evolution of the nuclei, we performed additional simulations where the two dynamical effects due to MBHs mentioned above were not included. (Hereafter we refer to these models as models "without MBH heating.") The blue and red curves in the middle panels of Figure 3 show the scaling correlations resulting from such models. When compared to our fiducial models (upper panels), these new integrations produce steeper and significantly tighter $M_{\mathrm{NSC}}-\sigma$ and $M_{\mathrm{NSC}}-M_{\mathrm{gx}}$ relations. This indicates that the break in the NSC scaling relations, for which we found evidence in the observational data, can be attributed to the interaction of NSCs with their companion MBHs.

Simple arguments can indeed be used to understand why the break of the NSC scaling relations occurs at $\sigma \approx 100 \mathrm{~km} \mathrm{~s}^{-1}$. From Equation (38), by requiring $r_{\text {disr }} \gtrsim r_{\mathrm{NSC}} \approx 10 \mathrm{pc}$ and adopting $r_{K}=1 \mathrm{pc}$ and $\sigma_{K}=20 \mathrm{~km} \mathrm{~s}^{-1}$, we find that MBHs more massive than roughly $\approx 10^{8} M_{\odot}$ will suppress the further growth of an NSC via accretion of stellar clusters. Thus, as the $\mathrm{MBH}$ grows, the contribution of star cluster inspirals to NSC growth decreases and eventually stops for $M_{\mathrm{MBH}} \gtrsim 10^{8} M_{\odot}$. Using (Ferrarese \& Ford 2005; Ferrarese et al. 2006)

$$
M_{\mathrm{MBH}} \approx 8.6 \times 10^{6}\left(\frac{\sigma}{100 \mathrm{~km} \mathrm{~s}^{-1}}\right)^{4.41} M_{\odot},
$$

an $\mathrm{MBH}$ mass larger than $\sim 10^{8} M_{\odot}$ corresponds to stellar spheroids with velocity dispersion $\sigma \gtrsim 150 \mathrm{~km} \mathrm{~s}^{-1}$. This is consistent with the value of $\sigma$ at which the NSC-host galaxy scaling correlations appear to flatten in the data sample.

Another argument leads us to identify $M_{\mathrm{MBH}} \approx 10^{8} M_{\odot}$ as the critical value of $\mathrm{MBH}$ mass above which NSCs are significantly affected. The impact of an $\mathrm{MBH}$ binary on a central cluster depends on the $M_{\mathrm{NSC}} / M_{\mathrm{MBH}}$ mass ratio. Given that during a merger an $\mathrm{MBH}$ binary will eject a mass comparable to its total mass, a requirement for an $\mathrm{MBH}$ binary to affect significantly the nuclear structure of a galaxy is that its mass is of the order of or larger than the mass of the surrounding cluster, i.e., $M_{\mathrm{MBH}} / M_{\mathrm{NSC}} \gtrsim 1$.

Using a standard weighted linear regression on our data gives the relation $\sigma \approx 50 \mathrm{~km} \mathrm{~s}^{-1}\left(M_{\mathrm{NSC}} / 10^{7} M_{\odot}\right)^{1.6}$; using
Equation (39), we find the new relation:

$$
\frac{M_{\mathrm{MBH}}}{6 \times 10^{7} M_{\odot}} \approx\left(\frac{M_{\mathrm{NSC}}}{6 \times 10^{7} M_{\odot}}\right)^{2.7} .
$$

Thus, the mass of the $\mathrm{MBH}$ grows faster than the mass of the NSC, with the transition from NSC-dominated to $\mathrm{MBH}$ dominated galaxies occurring at $M_{\mathrm{MBH}} \approx 10^{8} M_{\odot}$ or $\sigma \approx 150 \mathrm{~km} \mathrm{~s}^{-1}$. This is roughly the value of $\sigma$ at which NSCs appear to be significantly affected by their host galaxy MBHs in our models.

The total mass ejected from the center depends also on the number of stages in the merger hierarchy that have occurred since the MBHs first formed-i.e., after $N_{\mathrm{m}}$ mergers, the mass deficit is $\sim 0.7 N_{\mathrm{m}} M_{\mathrm{MBH}}$, with $M_{\mathrm{MBH}}$ the final (current) black hole mass (Merritt 2006). Some low-mass galaxies today might have never experienced a major merger. By contrast, more massive galaxies form via mergers of primordial lower-mass halos and underwent an above average number of mergers. In addition, since the occupation fraction of MBHs increases with galaxy mass, the scouring effect of $\mathrm{MBH}$ binaries is enhanced in the higher-mass spheroids.

From Figure 3, we also see that the $G x e V$ model generates two distinct populations of NSCs at $\sigma \gtrsim 100 \mathrm{~km} \mathrm{~s}^{-1}$, which can be easily identified by looking at the model confidence bands. Nuclei with $M_{\mathrm{NSC}} \lesssim 10^{7} M_{\odot}$ have masses that do not strongly correlate with their host galaxy mass and velocity dispersion - the mass of these NSCs lies significantly below the mass that we would obtain by extrapolating the scaling correlations from low to high galaxy masses. This population of underweight nuclei is a result of the disruptive MBH binary mergers that have partially eroded the surrounding clusters. NSCs above the model median seem instead to follow the general trend outlined in the low-mass galaxy region of the plot, where $M_{\mathrm{NSC}} \sim \sigma^{2}$. The host galaxies of such nuclei did not experience a major merger event since the epoch of the last major gas accretion event, so that their NSCs remained essentially unaffected by $\mathrm{MBH}$ binaries up to the present epoch. This picture is in agreement with expectations based on hierarchical models of galaxy formation-for example, Fakhouri et al. (2010) find that for a dark halo mass of $10^{12} M_{\odot}$ only $31 \%, 53 \%$, and $69 \%$ of these halos have experienced a major merger since $z=1,2$, and 3, respectively.

We can now ask which of the two processes, tidal disruption of star clusters or mass ejection from $\mathrm{MBH}$ binaries, is responsible for the broadening of the scaling correlations found in the data.

We find that both $C l i N$ models with $M_{\mathrm{ej}}=0$ and ones without the tidal truncation/disruption of clusters by the central $\mathrm{MBH}$ produce scaling correlations that appear to flatten at $\sigma \gtrsim 100 \mathrm{~km} \mathrm{~s}^{-1}$. The NSC scaling correlations produced by GxeV models in which we set $M_{\mathrm{ej}}=0$ (but in which we included the tidal disruption of migrating clusters) are instead at odds with observations, as they show no broadening of the NSC host galaxy property correlations for high velocity dispersion galaxies; only GxeV models that do take into account the scouring effect of $\mathrm{MBH}$ binaries were found to be in good agreement with the observed relations. We conclude that in $G x e V$ the crucial ingredient to reproduce the $M_{\mathrm{NSC}}-$ host galaxy property scaling relations is the scouring effect of $\mathrm{MBH}$ binaries, while the tidal stripping of migrating clusters by central MBHs is only a secondary effect in these models. We 


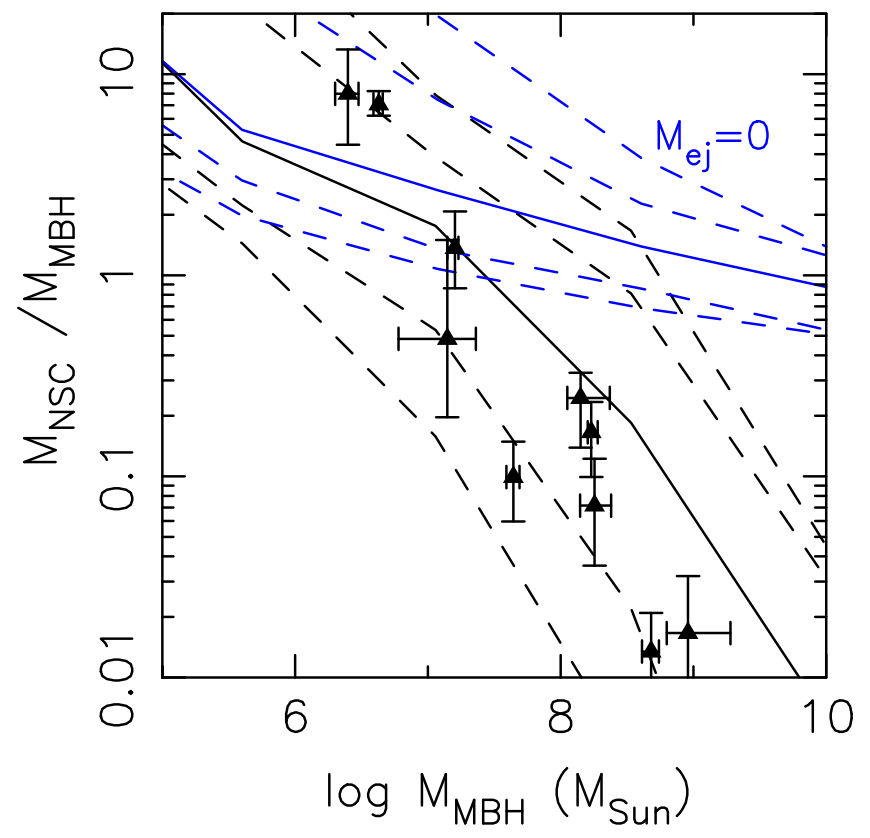

Figure 5. Fraction of NSC to MBH mass as a function of MBH mass. This plot measures the relative importance of the two types of central objects as one proceeds from NSC-dominated to $\mathrm{MBH}$-dominated nuclei. The points are observational data and represent galaxies with measured MBH and NSC mass (Graham \& Spitler 2009; Erwin \& Gadotti 2012). The black curve and corresponding $70 \%$ and $90 \%$ confidence bands are obtained through our fiducial galaxy formation model, where all the relevant effects described in Section 2.2 were included. The blue lines correspond to a model in which the scouring effect of MBH binaries forming during galaxy mergers was ignored, i.e., we set $M_{\mathrm{ej}}=0$ in these integrations. Note how the predictions of the two models start to diverge at $M_{\mathrm{MBH}} \approx 10^{8} M_{\odot}$, where MBHs and NSCs have comparable masses in the model with $M_{\mathrm{ej}}=0$; at $M_{\mathrm{MBH}} \gtrsim 10^{8} M_{M_{\odot}} \mathrm{MBH}$ mergers become efficient at eroding the surrounding NSC.

remark that the $\mathrm{GxeV}$ model has a clear advantage over the CliN model, because the former follows the hierarchical evolution of NSCs and MBHs along merger trees, while the latter assumes a monolithic evolution. Although the results of the two models are generally in good agreement with one another, the differences outlined above are mainly a result of the implicit assumption made in the CliN model that the NSCs always grow around preexisting MBHs. This leads to an artificially enhanced mass removal from the stellar clusters accreting onto the central NSC, because of the MBH tidal field.

To illustrate more clearly the role of $\mathrm{MBH}$ binaries in shaping their companion NSC properties, we compare in Figure 5 the observationally constrained NSC-to-MBH mass ratio in galaxies containing both types of central objects to the same ratio obtained in (i) our fiducial $\mathrm{GxeV}$ model containing all the relevant effects described in Section 2.2, and (ii) a model in which we set $M_{\mathrm{ej}}=0$, i.e., in which the scouring effect of MBH binaries was not included in the calculation. Clearly, the scouring effect due to MBH binaries described by Equations (34) and (36) is the crucial ingredient to reproduce the observed correlation. Also, note that the correlations produced by the two models start to diverge from each other at $M_{\mathrm{MBH}} \approx 10^{8} M_{\odot}$, in agreement with our predictions (see also Antonini et al. 2015).

\subsubsection{NSC and MBH Occupation Fraction}

Observationally, the frequency of nucleation in early-type galaxies is found to increase sharply from zero for spheroids

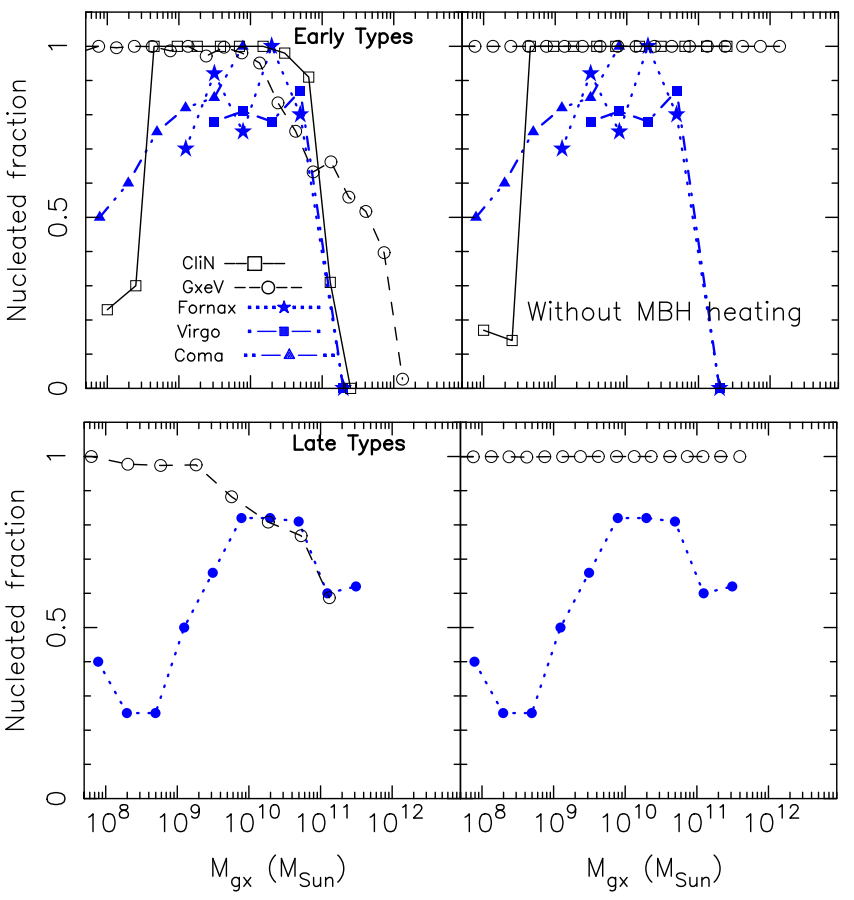

Figure 6. Top: local fraction of nucleated galaxies of our models compared to observational constraints on the nucleated fraction of early-type galaxies in Virgo (Côté et al. 2006), Fornax (Turner et al. 2012), and Coma (den Brok et al. 2014). The bottom panels are for late-type galaxies. The left panels show the results of our fiducial models with all the relevant effects included; the right panels correspond to models with no dynamical heating due to MBHs (see text for details). A comparison between the latter models and our fiducial models indicates that the lack of NSCs in galaxies more massive than $\approx 10^{11} M_{\odot}$ is due to MBHs that fully destroy the surrounding clusters during galaxy mergers and also quench their growth by disrupting migrating clusters owing to their strong central tidal field.

brighter than $M_{B}=-19.5$ to $\gtrsim 90 \%$ for galaxies fainter than this magnitude (Côté et al. 2006; Turner et al. 2012). In a sample of 332 late-type galaxies, Georgiev \& Böker (2014) found that $\gtrsim 80 \%$ of these galaxies harbor a well-defined NSC. Hence, NSCs are found in most galaxies of all Hubble type, but tend to disappear in the brightest spheroids.

Figure 6 compares the frequency of nucleation obtained in our models to that of late- and early-type galaxies, as derived from observations. We define here early-type galaxies as systems with bulge-to-total mass ratio $M_{\text {bulge }} / M_{\mathrm{gx}} \gtrsim 0.7$, and late-type galaxies as systems with $M_{\text {bulge }} / M_{\mathrm{gx}} \lesssim 0.7$. In agreement with observations, our models predict that almost all early-type galaxies that are less massive than $\approx 10^{11} M_{\odot}$ contain an NSC, and that the frequency of nucleation is nearly zero for galaxies with mass larger than this value. We note that the exact occupation number obtained through $\mathrm{GxeV}$ should be considered somewhat approximate-the identification of a galaxy as nucleated in this model is uncertain given that we cannot directly reconstruct the density profile of the NSC and compare it to the density profile of the galaxy background. Nevertheless, the occupation fraction is found to decrease significantly for early-type galaxies with mass larger than $10^{11}$ $M_{\odot}$, in good agreement with observational constraints. The bottom panel of Figure 6 shows the frequency of nucleation in late-type systems. Approximately $90 \%$ of these galaxies were found to contain an NSC, also in fairly good agreement with observations. 

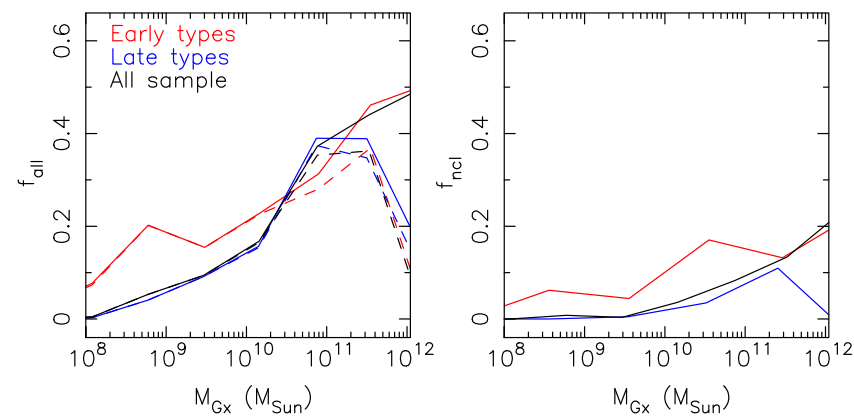

Figure 7. Left: predicted fraction of all galaxies, $f_{\text {all }}$, containing an $\mathrm{MBH}$ ( $M_{\mathrm{MBH}} \gtrsim 10^{5} M_{\odot}$; solid lines) and those containing an MBH plus an NSC (dashed lines). Right: fraction of nucleated galaxies, $f_{\text {ncl }}$, that contain a central AGN, identified here as an $\mathrm{MBH}$ with bolometric luminosity $L>10^{10} L_{\odot}$.

The observed drop in the nucleation frequency at high galaxy masses is often attributed to the disruptive effects of $\mathrm{MBH}$ binaries forming during galaxy mergers (Bekki \& Graham 2010) and to the tidal disruption of migrating clusters at large galactocentric distances by central MBHs (Antonini 2013). In the right panels of Figure 6 we show the nucleated fraction in models where the dynamical heating due to MBHs on migrating clusters and during mergers was not included. In these models the percentage of nucleation remains close to $100 \%$ regardless of galaxy luminosity, demonstrating that $\mathrm{MBHs}$ are responsible for the absence of NSCs in the most luminous galaxies, as also argued in previous work.

NSCs and MBHs are known to coexist in some galaxies across a wide range of masses and Hubble types (Seth et al. 2008b). However, not all NSCs contain an MBH; an example is the M33 NSC that has no central MBH down to highly constraining limits (Gebhardt et al. 2001; Merritt et al. 2001). Studies indicate that at least some NSCs can host a central $\mathrm{MBH}$, but the overall occupation fraction of MBHs in NSCs remains unknown. The left panel of Figure 7 displays the fraction of all galaxies in the GxeV model that contain an $\mathrm{MBH}$ and the fraction of galaxies containing both an $\mathrm{MBH}$ and an NSC. Our models predict that a high fraction of galaxies of intermediate and low luminosity contain a central NSC, but that the NSCs tend to disappear in massive galaxies. Thus, the total fraction of mixed systems with both an NSC and an $\mathrm{MBH}$ is roughly equal to the $\mathrm{MBH}$ occupation fraction for galaxies with mass $M_{\mathrm{gx}} \lesssim 10^{11} M_{\odot}$ and is roughly equal to the NSC occupation fraction for galaxies more massive than $10^{11} M_{\odot}$. In the right panel of Figure 7 we show the fraction of nucleated galaxies that contain an $\mathrm{MBH}$ with bolometric luminosity $L>10{ }^{10} L_{\odot}$. We find that the fraction of galaxies with an NSC that also have an active $\mathrm{MBH}$ increases from $\sim 5 \%$ to $30 \%$ going from $M_{\mathrm{gx}} \sim 10^{9} M_{\odot}$ to $\sim 10^{12} M_{\odot}$. These results are in fairly good agreement with the observational results from Seth et al. (2008b), who found that about $10 \%$ of NSCs in their spectroscopic sample also host an AGN.

\subsection{In Situ versus Migration: The Relative Contribution to NSC Growth}

The bottom panels of Figure 3 show the scaling relations generated by a version of the $G x e V$ model with no contribution from cluster inspirals. In these models, the only mechanism responsible for the formation and growth of the nuclei is local fragmentation of gas that leads to star formation and in situ buildup of a central compact cluster.
The bottom panels of Figure 3 demonstrate that even a purely dissipative model provides a quite good description of the observed NSC-host galaxy scaling relations. The median of the mass distribution as a function of $\sigma$ can be fit by $M_{\mathrm{NSC}} \approx 6.5 \times 10^{6}(\sigma / 100)^{1.4} M_{\odot}$. Hence, in this model we obtain scaling relations that are consistent with the observation that NSCs follow scaling relations that are shallower than the corresponding ones for MBHs.

We can derive analytical scaling relations for the in situ formation model by considering the $\mathrm{MBH}$ and reservoir characteristic timescales of evolution. Let us consider a situation in which the $\mathrm{MBH}$ is hosted by a gas-rich nucleus (resulting from a recent starburst; see Equation (24)), i.e., $M_{\mathrm{MBH}} \ll M_{\text {res }}$. The $\mathrm{MBH}$ will then grow approximately at the Eddington rate, i.e., $\dot{M}_{\mathrm{MBH}} \approx M_{\mathrm{MBH}} / t_{\text {salp }}$, with $t_{\text {salp }}$ the Salpeter timescale

$$
t_{\mathrm{salp}}=\frac{k \epsilon c}{4 \pi G}=4.5 \times 10^{7}\left(\frac{\epsilon}{0.1}\right) \mathrm{yr} .
$$

where $\epsilon$ is the radiative efficiency and $k \equiv 0.398 \mathrm{~cm}^{2} \mathrm{~g}^{-1}$ the electron scattering opacity. Gas accumulates at the center of the galaxy mainly during starbursts (see Equation (24)). Bursts of star formation will take place on the dynamical timescale of the galactic bulge, i.e.,

$$
t_{\mathrm{dyn}}=\frac{R}{\sigma}=10^{7}\left(\frac{\sigma}{100 \mathrm{~km} \mathrm{~s}^{-1}}\right)^{2.06} \text { years, }
$$

with $R$ the galactic bulge scale radius. In the last expression we have used the fact that both $R$ and $\sigma$ are related to the total galaxy luminosity through the empirical correlations (Bernardi et al. 2003; Dekel \& Cox 2006):

$$
R \approx 2.6\left(\frac{L}{1.6 \times 10^{10} L_{\odot}}\right)^{0.7} \mathrm{kpc}
$$

and

$$
\sigma \approx 150\left(\frac{L}{1.6 \times 10^{10} L_{\odot}}\right)^{0.23} \mathrm{~km} \mathrm{~s}^{-1}
$$

We can thus write, from Equation (24), $\dot{M}_{\text {res }} \approx A_{\text {res }} M_{\text {bulge }}^{\text {gas }} / t_{\text {dyn }}$ during the starburst event, and therefore

$$
\frac{\Delta M_{\mathrm{res}}}{\Delta M_{\mathrm{MBH}}} \approx \frac{\dot{M}_{\mathrm{res}}}{\dot{M}_{\mathrm{MBH}}} \approx \frac{t_{\mathrm{salp}}}{t_{\mathrm{dyn}}} \frac{A_{\mathrm{res}} \Delta M_{\mathrm{bulge}}^{\star}}{M_{\mathrm{MBH}}},
$$

where $\Delta M_{\text {res }}$ is the total mass of cold gas that falls to the nucleus during the star formation event, and $\Delta M_{\mathrm{MBH}}$ and $\Delta M_{\text {bulge }}^{\star}$ denote the changes in the $\mathrm{MBH}$ and stellar bulge masses during the starburst. (Note that eventually all the gas of the bulge is transformed into stars, if feedback is neglected, i.e., $M_{\text {bulge }}^{\text {gas }} \approx \Delta M_{\text {bulge. }}^{\star}$ )

Now, if we assume that the MBH approximately satisfies the Magorrian relation (Magorrian et al. 1998; Häring \& Rix 2004) $M_{\mathrm{MBH}} \approx A_{\mathrm{M}} M_{\text {bulge }}^{\star}$ (with $A_{\mathrm{M}} \approx 1.4 \times 10^{-3}$; Häring \& Rix 2004), we can write

$$
\frac{\Delta M_{\mathrm{res}}}{\Delta M_{\mathrm{MBH}}} \approx \frac{t_{\mathrm{salp}}}{t_{\mathrm{dyn}}} \frac{A_{\text {res }} \Delta M_{\text {bulge }}^{\star}}{A_{\mathrm{M}} M_{\text {bulge }}^{\star}} \approx \frac{t_{\text {salp }}}{t_{\text {dyn }}} \frac{A_{\text {res }}}{A_{\mathrm{M}}} f_{\text {crit }} \propto \sigma^{-2.06},
$$

where we have assumed $\Delta M_{\text {bulge }}^{\star} / M_{\text {bulge }}^{\star} \approx f_{\text {crit }}$, because starbursts are typically triggered by major galaxy mergers, 


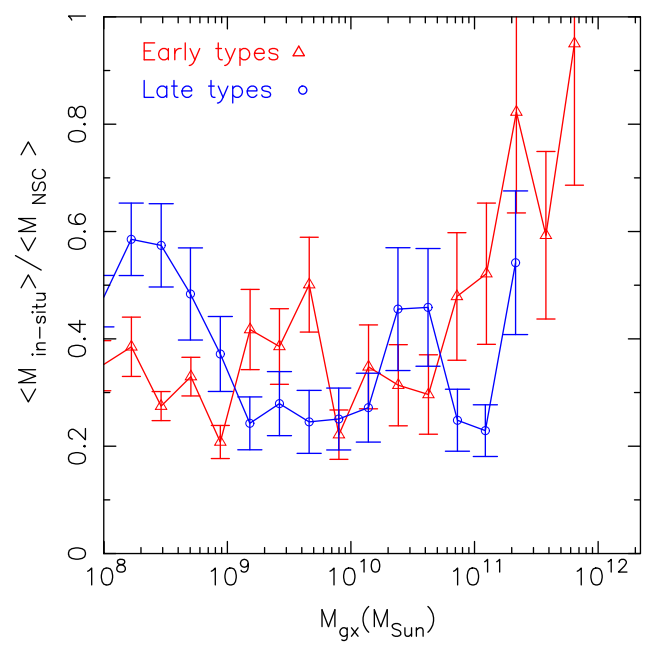

Figure 8. Ratio of the median mass obtained in the only in situ star formation model to the median mass of our fiducial model, which also includes cluster inspiral processes $\left(\left\langle M_{\text {insitu }}\right\rangle /\left\langle M_{\mathrm{NSC}}\right\rangle\right)$. Error bars represent $1 \sigma$ uncertainties. A large fraction of the mass of our model NSCs is built up locally from episodes of star formation triggered by infalling gas.

i.e., ones with mass ratio between the baryonic components of the two galaxies larger than $f_{\text {crit }} \approx 0.25$. ${ }^{9}$ Eventually, all the gas of the reservoir will either form stars or accrete onto the $\mathrm{MBH}$, i.e., $\Delta M_{\mathrm{res}}=\Delta M_{\mathrm{MBH}}+\Delta M_{\mathrm{NSC}}$. However, since we assumed $M_{\mathrm{MBH}} \ll M_{\text {res }}$, most of the nuclear gas will end up in the NSC rather than in the $\mathrm{MBH}$, hence $\Delta M_{\mathrm{res}} \approx \Delta M_{\mathrm{NSC}}$, and we can write, for $M_{\mathrm{MBH}} \ll M_{\mathrm{NSC}}$,

$$
\frac{d M_{\mathrm{NSC}}}{d \sigma} \approx \frac{t_{\mathrm{salp}}}{t_{\mathrm{dyn}}} \frac{A_{\mathrm{res}}}{A_{\mathrm{M}}} f_{\text {crit }} \frac{d M_{\mathrm{MBH}}}{d \sigma},
$$

where we used $\Delta M_{\mathrm{NSC}} \approx\left(d M_{\mathrm{NSC}} / d \sigma\right) \Delta \sigma \quad$ and $\Delta M_{\mathrm{MBH}} \approx\left(d M_{\mathrm{MBH}} / d \sigma\right) \Delta \sigma .^{10}$

We can then integrate this equation to give

$$
\begin{aligned}
M_{\mathrm{NSC}} & \approx 1.9 \times \frac{t_{\mathrm{salp}}}{t_{\mathrm{dyn}}} \frac{A_{\text {res }}}{A_{\mathrm{M}}} f_{\text {crit }} M_{\mathrm{MBH}} \\
& \approx 7.8 \times 10^{7} M_{\odot}\left(\frac{\sigma}{100 \mathrm{~km} \mathrm{~s}^{-1}}\right)^{2.35},
\end{aligned}
$$

where we have used Equation (39) and assumed a radiative accretion efficiency $\epsilon \approx 0.1$. Although very simplified, this model approximately reproduces the scaling relations generated by our dissipative $G x e V$ model (for $M_{\mathrm{MBH}} \lesssim M_{\mathrm{NSC}}$ ) in slope and also (within a factor of a few) in normalization.

The mass growth of NSCs is regulated by two processes: inspiral of star clusters and in situ star formation. In order to quantify the amount of NSC mass that is contributed by local star formation in our models, we computed the ratio of the median NSC mass obtained by including both cluster inspirals

\footnotetext{
$\overline{9}$ Note that the same approximate scaling $\Delta M_{\mathrm{res}} / \Delta M_{\mathrm{MBH}} \propto \sigma^{-2.06}$ can be obtained from Equation (45) by assuming that the MBH satisfies the $M-\sigma$ relation $M_{\mathrm{MBH}} \sim \sigma^{4}$, if one observes that $M_{\text {bulge }}^{\star} \sim\left(M_{\text {bulge }}^{\star} / L\right) L \sim \sigma^{4}$ (where we have used Equation (44) and assumed constant mass-to-light ratio). Indeed, the latter relation yields $\Delta M_{\text {bulge }}^{\star} \sim \sigma^{3} \Delta \sigma$, which replaced in Equation (45) gives $\Delta M_{\mathrm{res}} / \Delta M_{\mathrm{MBH}} \propto \sigma^{-2.06}$, if one assumes $\Delta \sigma \sim \sigma$ in major galactic mergers.

${ }^{10}$ Note that $d M_{\mathrm{MBH}} / d \sigma$ and $d M_{\mathrm{NSC}} / d \sigma$ are essentially the rates of change of the $\mathrm{MBH}$ and NSC masses during a starburst (time being parameterized by the host galaxy's velocity dispersion $\sigma$ ).
}

and in situ star formation to that obtained with only the contribution of in situ star formation. We plot this quantity as a function of total galaxy mass in Figure 8.

In our models, about half of the mass of NSCs is contributed by local star formation for galaxies less massive than $\sim 3 \times 10^{11} M_{\odot}$, while for galaxies more massive than this value, the contribution from dissipative processes becomes progressively more important. Some provisional evidence for a preferential dissipative mode in galaxies of progressively larger masses was presented by Côté et al. (2006) and Turner et al. (2012). These authors showed that the brightest nuclei that reside in the most luminous hosts have colors that are significantly redder than expected on the basis of a star cluster merger scenario, suggesting that an additional process-e.g., dissipational infall of metal-rich gas during mergers-likely begins to dominate the formation of nuclei in galaxies of higher masses.

We finally note that the relative contribution of the two formation channels depends on the adopted value of star cluster formation efficiency $f_{\mathrm{gc}}$, which remains a quite uncertain parameter of our models. However, given that plausible values of $f_{\mathrm{gc}}$ were found to only impact the normalization of our results for the empirical relations and to not affect their slope, assuming $f_{\mathrm{gc}} \approx$ const, we can simply express the relative contribution of the two formation channels using the general formulation:

$$
\frac{\left\langle M_{\mathrm{cl}}\right\rangle}{\left\langle M_{\mathrm{insitu}}\right\rangle} \approx 1.5 \times\left(\frac{f_{\mathrm{cl}}}{0.07}\right),
$$

with $\left\langle M_{\text {insitu }}\right\rangle$ the typical NSC mass that originates in situ from episodes of star formation and $\left\langle M_{\mathrm{cl}}\right\rangle$ the mass brought in by migrating clusters.

\section{COSMOLOGICAL EVOLUTION OF SCALING CORRELATIONS}

The existence of a fundamental connection between MBHs and NSCs was first suggested by Ferrarese et al. (2006). These authors showed that NSCs and MBHs follow similar scaling relations with their host galaxy properties, and thus argued that they are two different manifestations of the same astrophysical type of system, which they called "central massive object." Later, Graham (2012b) used a large sample of nucleated galaxies to show that the scaling relations of NSCs and MBHs are quite different from each other, with the former following much shallower correlations with their host galaxy properties. Graham's findings might suggest that NSCs and MBHs formed through different physical processes. This could be the case if, for example, NSCs originated elsewhere in the galaxy and then migrated to the center through dynamical friction processes (e.g., Antonini 2013).

Neumayer \& Walcher (2012) presented a first $M_{\mathrm{MBH}}$ versus $M_{\mathrm{NSC}}$ diagram and found, in agreement with our study, the existence of three different regimes: (a) NSC-dominated nuclei at $\sigma \lesssim 100 \mathrm{~km} \mathrm{~s}^{-1}$, (b) a transition region, and (c) $\mathrm{MBH}$ dominated nuclei at $\sigma \gtrsim 150 \mathrm{~km} \mathrm{~s}^{-1}$. Neumayer \& Walcher (2012) argued that this is consistent with a picture in which black holes form inside NSCs with a low mass fraction. They subsequently grow much faster than the NSC, destroying it completely when the ratio $M_{\mathrm{MBH}} / M_{\mathrm{NSC}}$ grows above $\sim 100$.

Neumayer \& Walcher (2012) also argued against $\mathrm{MBH}$ mergers as responsible for the disruption of the nuclei in the 
highest-mass galaxies. These authors pointed out that if NSCs are disrupted during mergers, elliptical galaxies-thought to be the product of galaxy mergers-should rarely host an NSC. The fact that most early-type galaxies have an NSC would therefore suggest that mergers do not play a pivotal role in leading to NSC disruption. We do not agree with this interpretation.

As discussed in Section 4.1, an MBH binary will eject from the galaxy center a mass comparable to its own mass, so that only mergers with $\mathrm{MBH}$-to-NSC mass ratio larger than unity can significantly heat the NSC and make it susceptible to destruction during the merger event. NSCs can therefore survive and indeed grow during the morphological transformation from disk-dominated to bulge-dominated galaxies if the progenitor galaxy NSCs are significantly more massive than their central MBHs. Indeed, from the NSC and MBH scaling correlations, we find that NSCs dominate the nuclei of galaxies with velocity dispersion $\sigma \lesssim 150 \mathrm{~km} \mathrm{~s}^{-1}$, so that mergers of galaxies at the low end of the $\sigma$ distribution will not lead to the complete disruption of the host galaxy NSCs, while mergers of massive galaxies, characterized by a larger MBH-to-NSC mass ratio, will lead to their full disruption. This picture is consistent with both the abundance of NSCs in early-type galaxies of intermediate luminosity and the fact that NSCs tend to disappear in the brightest spheroids hosting the most massive MBHs.

To illustrate the simultaneous evolution of MBHs and NSCs in our semi-analytical galaxy formation models, we plot in Figure 9 the MBH and NSC scaling correlations at different redshifts and the corresponding mass distributions in Figure 10. Specifically, in order to calculate the median and confidence regions in our model, we have have only considered the MBHs residing in bulge-dominated galaxies (which we identify with ones having bulge-to-total mass ratio larger than 0.7). ${ }^{11}$

We find that at high redshifts, $z \gtrsim 3$, the nuclei of galaxies are dominated by NSCs. Between redshift 4 and 2 the MBHs grow faster than the NSCs, becoming by $z \approx 3$ the dominant nuclear component in galaxies with $\sigma \gtrsim 100 \mathrm{~km} \mathrm{~s}^{-1}$. After this point, the NSC scaling correlations start to flatten at high values of $\sigma$, as $\mathrm{MBH}$ binaries forming during mergers are now efficient at eroding the surrounding clusters. In addition, the NSCs can no longer grow efficiently in the most massive galaxies, since inspiraling stellar clusters are tidally disrupted at larger galactocentric distances in galaxies with progressively more massive MBHs.

Figure 10 displays the redshift evolution of the mass distribution of MBHs and NSCs. In these plots we include all galaxies in our models regardless of their specific value of bulge-to-total mass ratio. At high redshift the NSCs are typically more massive than MBHs; after $z \approx 2$ the mass distribution of NSCs intersects at about $10^{8} M_{\odot}$ the distribution of MBHs. This sets the transition between NSC-dominated and MBH-dominated galaxies seen also in the observational data. We also compared our synthetic NSC mass distributions for early-type galaxies with the observed mass distribution of NSCs for the catalogs of early-type galaxies of Côté et al. (2006) and Turner et al. (2012). Anderson-Darling and Kolmogorov-Smirnov statistical tests used to compare the observed to the MBH model cumulative distributions give $p$ -

\footnotetext{
11 We make this choice to correct for the observational bias that selects galaxies with a significant bulge component, so that measurements of $\sigma$ are possible in the first place.
}
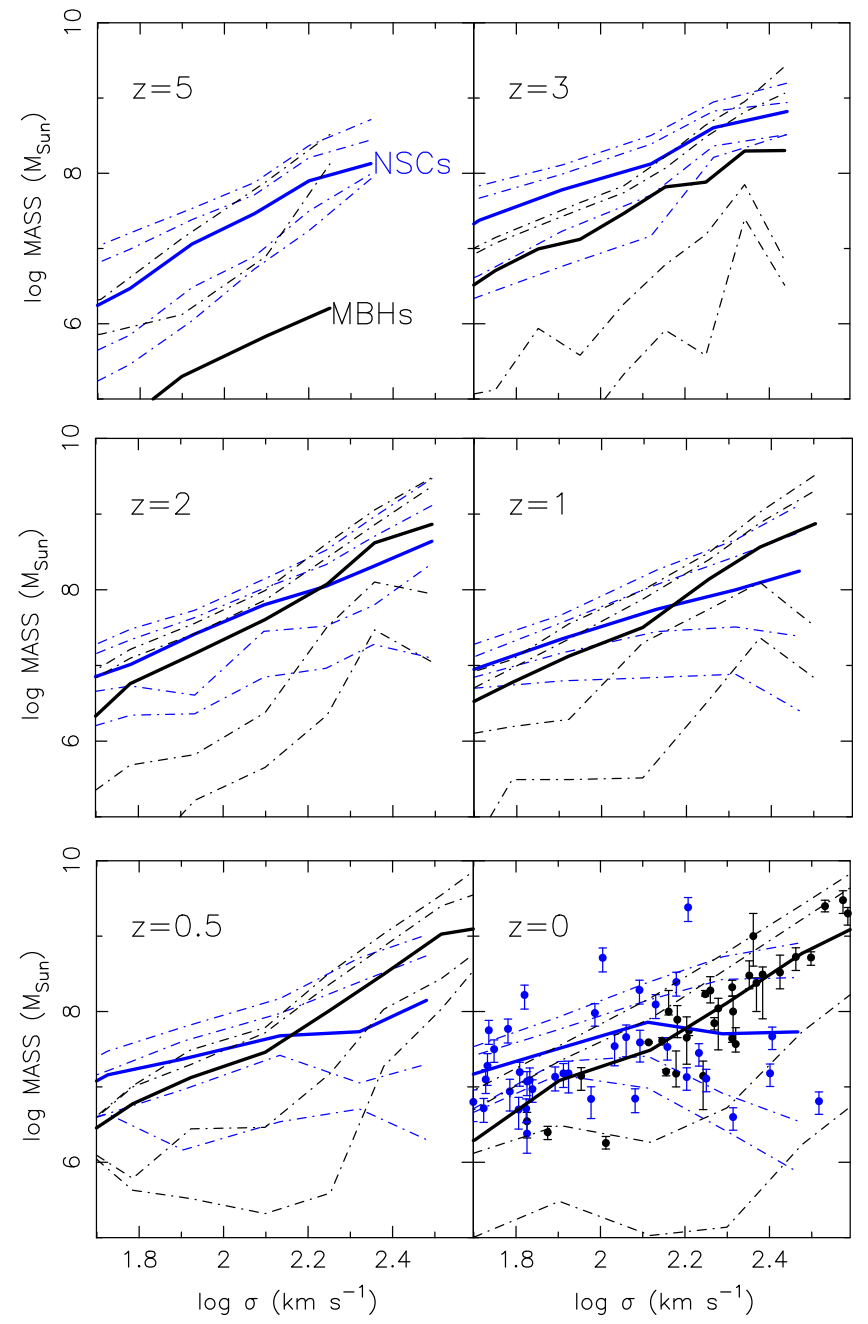

Figure 9. Scaling correlations of NSCs and MBHs in our galaxy formation model at different redshifts. At high redshift the NSCs are the dominant central component of galaxies; between $z=3$ and $z=1$ the MBHs grow faster, and by $z=2$ they are the dominant central component in galaxies with $\sigma \gtrsim 100 \mathrm{~km} \mathrm{~s}^{-1}$. After this point, MBH mergers become efficient at carving out the preexisting nuclei. This effect induces the bending/broadening of the $\mathrm{M}_{\mathrm{NSC}}-\sigma$ relation toward high galaxy masses at low redshifts, which can be clearly seen in the bottom panels of the figure. In the bottom right panel, blue points represent NSCs and black points represent MBHs (Tremaine et al. 2002)

values of 0.35 and 0.42 , respectively, indicating that the hypothesis that the simulated and observed distributions are significantly different from each other can be rejected at a high level of confidence. For a comparison with the observed MBH mass function at $z=0$, see instead Barausse (2012) and Sesana et al. (2014).

\subsection{Dependence on the MBH Seed Model}

In the previous sections, we have discussed the results of a galaxy formation model in which MBHs are evolved starting at $z \sim 15-20$ from initial "light" masses $M_{\text {seed }} \sim 200 M_{\odot}$. This could be the case if MBHs formed as remnants of Population III stars (Madau \& Rees 2001).

In order to test the dependence of our results on the assumed scenario for the formation of MBHs, we evolved additional models in which halos are initially populated by MBH seeds of mass $M_{\text {seed }} \sim 10^{5} M_{\odot}$, which could, for example, form as a 


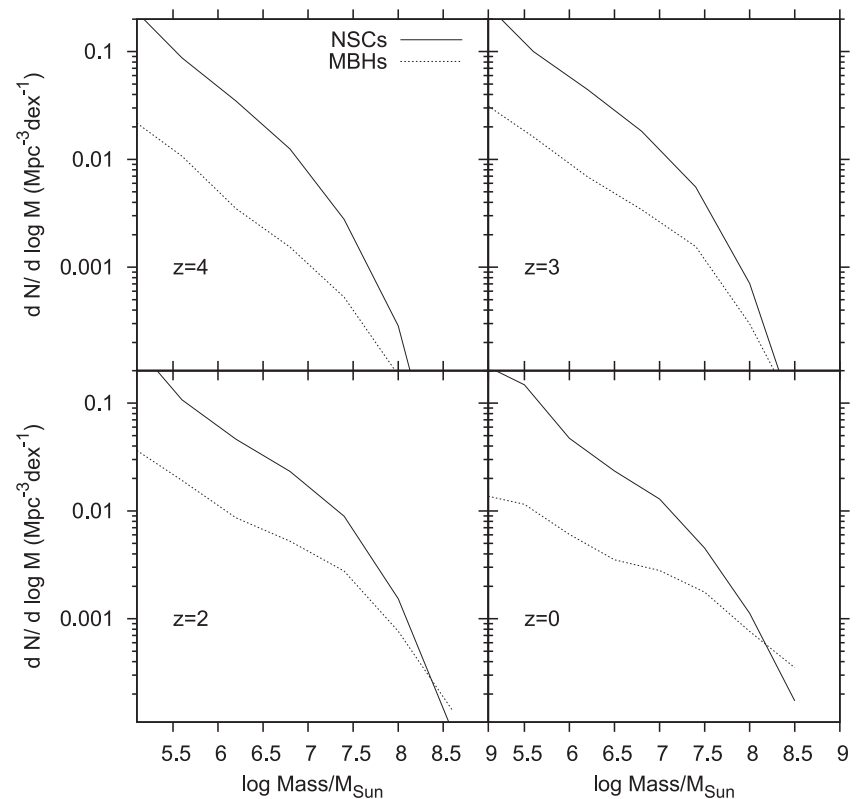

Figure 10. Mass distribution of MBHs and NSCs at various redshifts. At high redshift, $z \lesssim 4$, the NSCs are typically more massive than MBHs. At $z=0$ NSC masses are typically of the order $10^{6-7} M_{\odot}$, with only a few rare clusters having masses above $10^{8} M_{\odot}$. Note the transition from NSC-dominated to MBH-dominated galaxies for $M_{\mathrm{MBH}} \gtrsim 10^{8} M_{\odot}$ occurring at $z \lesssim 2$.

result of the collapse of massive protogalactic disks at redshifts $z \gtrsim 10-15$ (Koushiappas et al. 2004; Begelman et al. 2006; Lodato \& Natarajan 2006; Volonteri et al. 2008). In particular, we consider a model in which these "heavy" seeds form with high halo occupation number (Koushiappas et al. 2004), and one in which the halo occupation number is moderate (namely, the model of Volonteri et al. 2008, where we set the critical Toomre parameter below which the formation of an $\mathrm{MBH}$ seed becomes possible to $Q_{c}=2$ ).

Figure 11 shows the redshift evolution of the $M_{\mathrm{NSC}}-\sigma$ and $M_{\mathrm{MBH}}-\sigma$ relations in the case of the MBH heavy-seed scenario of Koushiappas et al. (2004). Figure 12 displays the corresponding $\mathrm{MBH}$ and NSC mass distributions from $z=4$ to $z=0$. A comparison of these plots with those in Figures 9 and 10 demonstrates that the local mass distribution of NSCs and their evolution from high redshift is not affected by the MBH seed model in any important way. (Of course, the MBH mass function in the heavy-seed models differs from that in the light-seed model at the low-mass end, which is dominated by MBHs that have not evolved significantly from their seeds. However, at intermediate and high masses, the MBH mass functions are very weakly dependent on the seed model, since memory of the initial conditions has been lost owing to accretion and mergers.) Note that also in the heavyseed scenarios, we find that the NSC cumulative mass distributions at $z=0$ for early-type galaxies are consistent with the observed mass distribution in Côté et al. (2006) and Turner et al. (2012) at a high level of significance (similar conclusions hold for the seed model of Volonteri et al. 2008).

The fact that our results about NSC evolution are not sensitive to the particular model chosen for the formation of MBH seeds is primarily a consequence of the high NSC-toMBH mass ratio at high redshift. At $z \gtrsim 3$, in both the heavyand light-seed scenarios, the NSCs are the dominant central component of galaxies (with the exception of small NSC
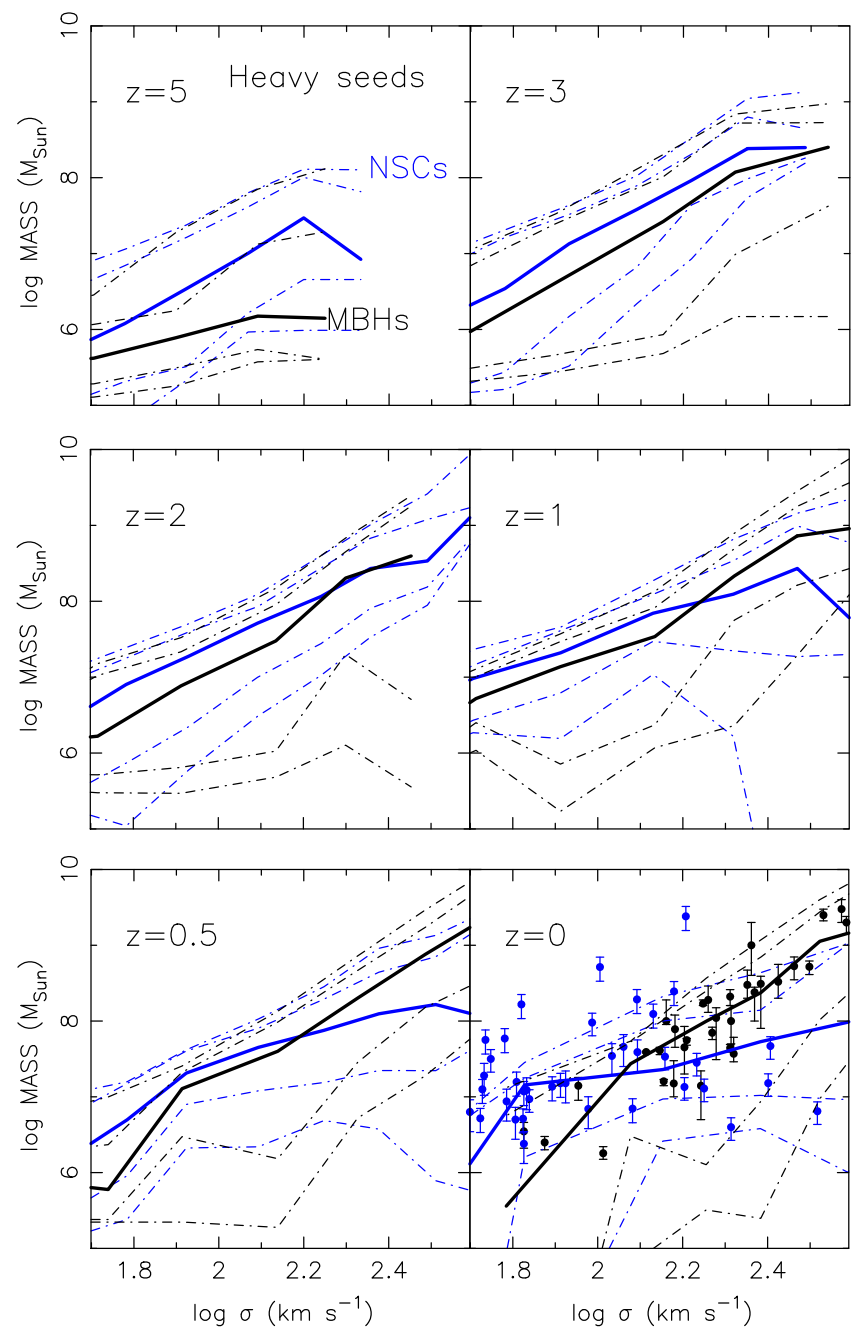

Figure 11. Same as Figure 9, but for the heavy MBH seed scenario of Koushiappas et al. (2004).

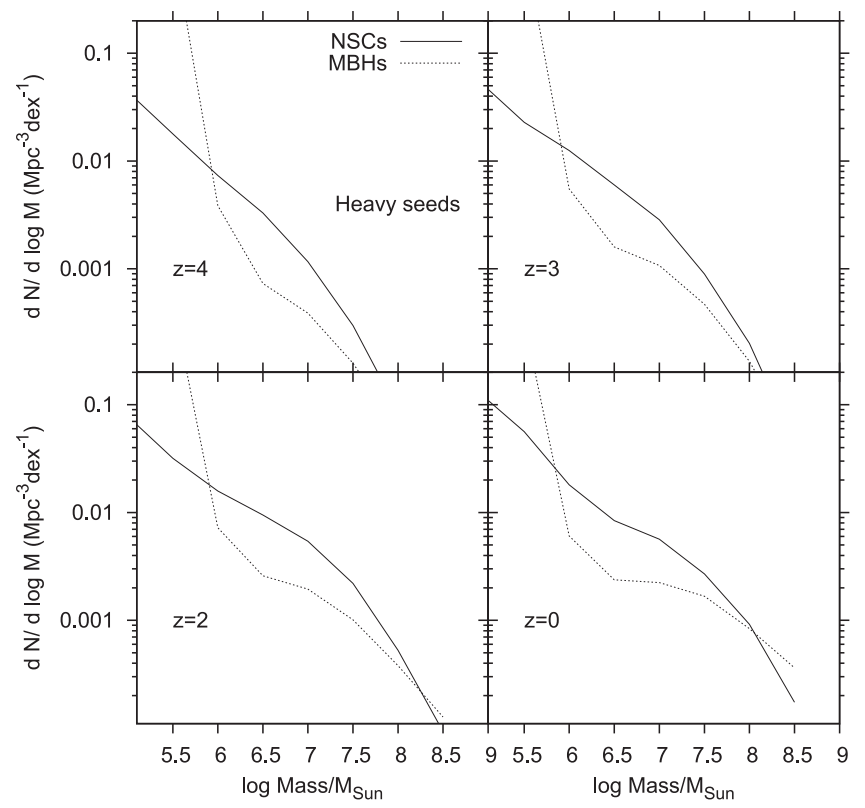

Figure 12. Same as Figure 10, but for the heavy MBH seed scenario of Koushiappas et al. (2004). 

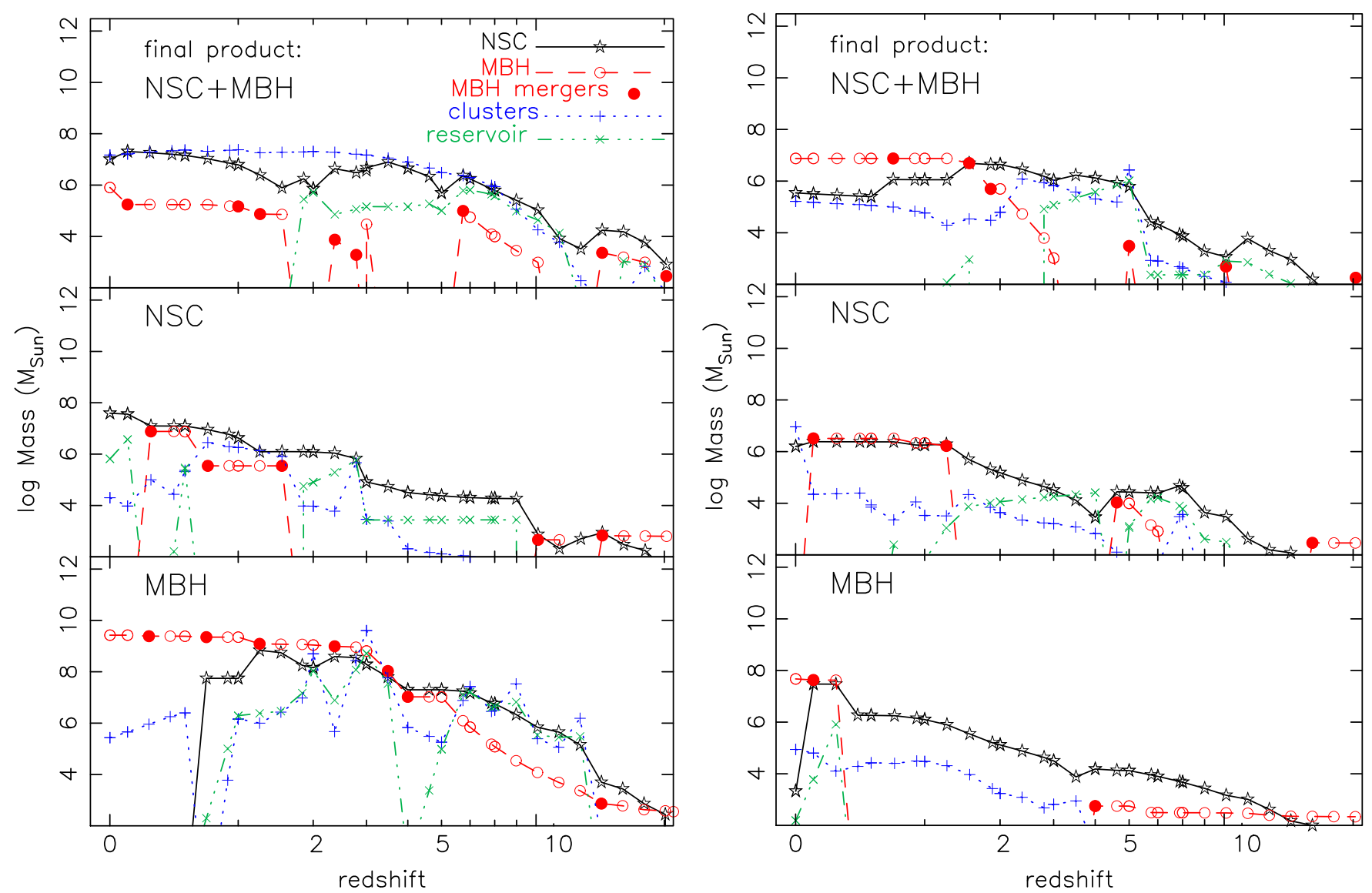

Figure 13. Examples of possible main-progenitor evolutions of NSCs and MBHs, as predicted by our model and for different final products at $z=0$. At upper left we indicate the final product of the evolution, a composite NSC $+\mathrm{MBH}$ nucleus (top panels), an NSC without MBH (middle panels), or an MBH-dominated nucleus lacking an NSC (bottom panels). We consider the evolution of the NSC hosted in the central galaxy at $z=0$ and proceed back in time, following the main NSC progenitor at each merger. However, when no NSC is present in the central galaxy at $z=0$ (namely, in the bottom left panel), we follow the MBH main-progenitor history.

masses $\lesssim 10^{6} M_{\odot}$, at which MBHs may dominate if they are seeded with high halo occupation number at high redshift). It is only after the peak of the quasar activity at $z \approx 2$ that MBHs become massive enough to significantly affect the NSCs at intermediate and high masses. However, by this time the $\mathrm{MBH}$ mass distributions in different seed scenarios are very similar to each other (again, with the exception of the low-mass end), and therefore the subsequent evolution of the NSCs is also very similar. The mass growth of the MBH population is in fact dominated by the mass accreted during the quasar epoch at $z \approx$ 2 , thus washing out the imprint of the initial conditions (see also Barausse 2012).

Since to date the formation process of MBHs remains largely unconstrained, the fact that our results are robust against different initial seed formation scenarios is important if we want to make robust predictions about the overall evolution of the NSC population.

\section{DISCUSSION}

\subsection{Formation of the Central Regions of Galaxies}

In this paper, we have presented a study aimed at understanding how the central regions of galaxies formed, and how the evolution of MBHs and NSCs is connected to that of their host galaxy.
In Figure 13, we show examples of NSC and $\mathrm{MBH}$ evolution predicted by the $G x e V$ model. These cases should be seen as qualitative because of the great variety of possible NSC and MBH histories that are possible within our model, but we have chosen examples that are roughly representative of the various possibilities mentioned below. In more detail, we consider the evolution of NSCs back in time following their main progenitor, i.e., we start from the NSC hosted in the central galaxy at $z=0$, and at each galaxy merger we follow its more massive NSC progenitor. When no NSC is present at $z=0$ in the central galaxy (namely, in the bottom left panel), we follow the main-progenitor history of the $\mathrm{MBH}$. As can be seen, NSCs grow by the combined action of (i) stellar cluster infall, which happens continuously throughout their history but is particularly enhanced in starburst galaxies (see the blue line in Figure 13, which denotes the mass in stellar clusters, and which shows a smooth evolution with superimposed spikes due to starbursts); and (ii) in situ star formation, which takes places for the most part in starburst galaxies formed by major mergers (see the spikes in the mass of the low angular momentum reservoir available for nuclear star formation, i.e., the green line in Figure 13). Note also that MBH mergers have a prominent effect on the MBH and NSC history, not only because MBH binaries erode or even completely destroy the NSC if the MBHs are sufficiently massive, but also because the merger can eject the remnant MBH from the galactic nucleus, when the 
kick velocity imparted by the anisotropic gravitational-wave emission is larger than the escape velocity from the central parts of the galaxy. Note instead that the apparent "regrowth" of the MBH after an ejection, which can be seen in Figure 13, is simply a consequence of our choice of following the NSC main-progenitor history: at certain galaxy mergers, the galaxy containing the main NSC (but no $\mathrm{MBH}$ ) may merge with one carrying an $\mathrm{MBH}$.

Below we discuss in more detail how the evolution of the galaxy and its merger history can give rise to the variety of galactic central structures observed today.

\subsubsection{Nucleated galaxies containing an MBH (see upper panels of Figure 13).}

If the $\mathrm{MBH}$ mass does not grow above $\approx 10^{7} M_{\odot}$ throughout the evolution of the galaxy, the central NSC cannot be significantly eroded during galaxy mergers by inspiraling $\mathrm{MBH}$ binaries, because of the large NSC-to-MBH mass ratio. Moreover, stellar clusters will migrate to the center essentially undisturbed by the tidal field of the central $\mathrm{MBH}$.

If during the last merger event the $\mathrm{MBH}$ remnant is retained, the end product will be a galaxy containing both a central NSC and an MBH; an example of such a galaxy is the Milky Way, which hosts an $\mathrm{MBH}$ of mass $\approx 4 \times 10^{6} M_{\odot}$ (Ghez et al. 1998; Gillessen et al. 2009) and an NSC of mass $\approx 3 \times 10^{7} M_{\odot}$ (Feldmeier et al. 2014; Schödel et al. 2014). A handful of external galaxies are also known to contain both an NSC and an $\mathrm{MBH}$, which are often found to have comparable masses (Seth et al. 2008a). These galaxies lie near the transition region between MBH-dominated and NSC-dominated systems. As the MBHs of these galaxies grow, $\mathrm{MBH}$ mergers will partially destroy their host NSCs in the process (e.g., top right panel of Figure 13).

\subsubsection{Nucleated galaxies without an $\mathrm{MBH}$ (see middle panels of Figure 13).}

After a merger, an $\mathrm{MBH}$ can receive a "kick" due to gravitational recoil with velocities as large as a few thousand kilometers per second, which may eject the $\mathrm{MBH}$ from its host galaxy. The ejected $\mathrm{MBH}$ will carry a cluster of bound stars, a hypercompact stellar system that can appear similar in size and luminosity to star clusters or ultracompact dwarf galaxies (Merritt et al. 2009). If the ejected MBH has a mass $\lesssim 10^{7} M_{\odot}$, the NSC will remain virtually unaffected. If from that point on the galaxy evolves passively without experiencing mergers that might bring a new $\mathrm{MBH}$ to the center, the evolution's end product will be a nucleus containing an NSC but no MBH. Examples of such a type of evolution are illustrated in the middle panels of Figure 13. A similar end product can be attained if an $\mathrm{MBH}$ seed never formed and the galaxy evolved without experiencing major mergers during its entire evolution.

We note in passing that whether an $\mathrm{MBH}$ seed will form might depend on the properties of the central cluster at high redshift (Miller \& Davies 2012). If the accumulated cluster has a high velocity dispersion $\sigma \gtrsim 40 \mathrm{~km} \mathrm{~s}^{-1}$, this might seed the growth of a central $\mathrm{MBH}$ because kinematic heating from binary stars is insufficient to prevent complete core collapse, which might then lead to runaway mergers and the formation of a central $\mathrm{MBH}$ seed of $\sim 10^{3} M_{\odot}$. In galaxies hosting clusters with initial velocity dispersion less than $40 \mathrm{~km} \mathrm{~s}^{-1}$, binaries will prevent core collapse and consequently the formation of an $\mathrm{MBH}$ seed. Galaxies with clusters with initial velocity dispersions below this limit might therefore never form an $\mathrm{MBH}$. Such an evolutionary path might lead to the formation of galactic nuclei such as those of M33 or NGC 205, which lack an $\mathrm{MBH}$ and host a low velocity dispersion NSC (Gebhardt et al. 2001; Merritt et al. 2001).

\subsubsection{MBH-dominated galaxies without an NSC (see bottom panels of Figure 13).}

After the $\mathrm{MBH}$ mass grows above $\gtrsim 10^{8} M_{\odot}$, any major merger will be highly disruptive for a central NSC; $\mathrm{MBH}$ binaries forming during major mergers in this high $\mathrm{MBH}$ mass regime will carve out the galactic center of stars destroying a preexisting NSC, eventually producing a central mass deficit (Milosavljević \& Merritt 2001; Bekki \& Graham 2010).

The accretion of a nucleated dwarf galaxy by a low-density giant galaxy could bring an NSC to the center of the latter galaxy. However, Merritt \& Cruz (2001) showed that the secondary galaxy is disrupted during the merger by the giant galaxy $\mathrm{MBH}$ tidal field, producing a remnant with a central density that is only slightly higher than that of the giant galaxy initially. Moreover, as shown in Antonini (2013), after an NSC is disrupted in a dry merger, it will have a hard time regrowing, because the $\mathrm{MBH}$ tidal field is effective at disrupting migrating clusters. Thus, once the galactic MBH mass grows above $\gtrsim 10^{8} M_{\odot}$, the central NSC is likely to be disrupted and the central core is likely to be preserved during the subsequent evolution of the galaxy.

\subsubsection{Low-mass spheroids without an NSC.}

NSCs tend to disappear in galaxies fainter than $M_{V} \sim-12$ (van den Bergh 1986). While the purely dissipationless formation model CliN predicts that the NSC occupation fraction decreases substantially in low-mass systems in agreement with observations, the $G x \mathrm{eV}$ model results in a high occupation fraction of NSCs in low-mass galaxies, and it is therefore in tension with observational findings. We believe that the reason for this discrepancy is that discreteness effects are not accounted for in $\mathrm{GxeV}$ as argued in the following. In the latter model, we compute the flux of mass accreting onto the nucleus by averaging over the initial mass function of the stellar cluster population. In CliN, we simulate instead the inspiral of each of the formed clusters, drawing their masses from the same initial mass function. Because of the initial small number of stellar clusters in low-mass galaxies, and because of the steep power law that we adopt for their initial mass function, some of these galaxies will host no cluster that is massive enough to decay to the center before being disrupted in the process. This suggests that the lack of NSCs in spheroids less luminous than $M_{V} \sim-12$ is a consequence of the initial low number of massive clusters in these galaxies. Clearly, this effect cannot be accounted for in the $G x e V$ model, which does not follow the evolution of individual stellar clusters but rather the average mass infall to the nucleus.

\subsection{Galaxy Cores and Cusp Regeneration}

Our analysis focuses on the mass evolution of NSCs and ignores effects due to dynamical relaxation that can change the density profile of the clusters over time. We have shown that the merger history of galaxies after $z \approx 2$ plays a key role in 
determining the structure of NSCs observed today and their disappearance in the brightest galaxies. In our models, we did not account for relaxation processes that can rebuild an eroded cusp as stars diffuse into the MBH sphere of influence. Even if an NSC is fully disrupted during a merger, a stellar cusp of mass of order $10 \%$ the mass of the black hole and extending out to $1 / 10$ its sphere of influence will reform over the relaxation time (Merritt \& Szell 2006). Although cusp regrowth is not expected to have an important effect on the mass evolution of the NSCs, it is worth discussing it, as the ability of an $\mathrm{MBH}$ to shape a galactic nucleus and the observational consequences depend on whether cores are regenerated or preserved after mergers.

Bekki \& Graham (2010) performed $N$-body simulations to investigate the evolution of merging star clusters with seed black holes. Using black holes masses of 1\%-5\% their host NSC ones, they investigated how the binary coalescence dynamically heats and destroys the surrounding clusters. While Bekki \& Graham (2010) argued that this mechanism provides a pathway to explain the observed reduction in the nucleus-togalaxy stellar mass ratio as one proceeds from dwarf to giant elliptical galaxies, they did not discuss how their results would be affected by two-body relaxation after the $\mathrm{MBH}$ binary merges and between mergers.

During galaxy mergers, cores are carved out in the stellar distribution, with radii of order the influence radius of the massive binary. This makes NSC susceptible to disruption. On the other hand, if the supply of stars is continuously replenished, the damage to the NSC can be much smaller. Furthermore, even if a large core is formed, a stellar density cusp can regrow via energy exchanges between stars moving in the gravitational field of the $\mathrm{MBH}$ remnant. Black holes with masses of $1 \%-5 \%$ that of the NSC, such as those considered in Bekki \& Graham (2010), correspond to low-mass galaxies with a short $\left(\lesssim 10^{9}\right.$ yr) relaxation time. From Equation (40) one finds that such a low mass ratio corresponds to an $\mathrm{MBH}$ mass of $\approx 5 \times 10^{5} M_{\odot}$. Using Equation (39), this corresponds to a host stellar spheroid velocity dispersion of $\approx 50 \mathrm{~km} \mathrm{~s}^{-1}$. In such low-mass galaxies, the cusp will regenerate itself in about 1 Gyr from the infall of a second black hole, as collisional relaxation drives the stellar distribution toward its steady-state form (Merritt 2013, p.544). We conclude that in low-mass spheroids the effect of MBH binaries on the host NSC density distribution is expected to be minimal, and their imprint on the nuclear properties is expected to be erased after a short time.

The probability that a galaxy of the size of the Milky Way experienced a major merger (i.e., with mass ratio larger than 0.3 ) after $z=2$ is about 50\% (Fakhouri et al. 2010). Galaxies of such luminosities have relaxation times that are longer than the age of the galaxy, but which are short enough that two-body relaxation might have affected their central clusters. These galaxies might have nuclei that are still evolving toward their steady state after they were dynamically heated by an $\mathrm{MBH}$ binary. Thus, the results of our models imply that "undermassive" NSCs in galaxies with bulge mass $M_{\text {bulge }} \approx 10^{10} M_{\odot}$ might still carry an imprint of the merger history of their host galaxy, in the form of a density core that extends out to a small fraction of the central $\mathrm{MBH}$ influence radius. Whether these NSCs will turn out to harbor parsec-scale cores will be addressed in the future with high-resolution imaging, which will enable us to resolve the crowded stellar environment of NSCs (Gullieuszik et al. 2014).

\section{CONCLUSIONS}

NSCs and MBHs represent important central components of galaxies that bear witness to their formation history. Our aim has been to generate a numerical framework to better understand how the central structures of galaxies formed and how the evolution of MBHs and NSCs is connected to that of their host galaxies.

We have studied the evolution of NSCs in a cosmological context, by taking into account the growth of MBHs by merging subhalos containing both MBHs and NSCs. A semianalytical galaxy formation model is applied to follow the evolution of dark matter halos along merger trees, as well as that of the baryonic components. Specifically, the evolution of dark matter halos along the merger trees includes their baryonic components such as hot gas, stellar and gaseous bulges, and stellar and gaseous galactic disks. We study the simultaneous evolution of MBHs and NSCs in our galaxy formation model. The main results of our work are summarized as follows:

(1) The mass growth of NSCs is seen to be regulated by inspiral of star clusters, in situ star formation, and galaxy and $\mathrm{MBH}$ mergers. We have found that both cluster inspirals and in situ star formation contribute a significant fraction of the total mass of NSCs.

(2) We found that in situ star formation (as well as growth through migrating clusters) generates NSC-host galaxy scaling relations that are much shallower than the same correlations for MBHs, in agreement with observations.

(3) In our model, the merger history of galaxies after $z \sim 2$ plays a key role in determining the structure of the NSCs observed today. Core depletion due to gravitational slingshot of host galaxy stars by inspiralling MBHs forming during galaxy mergers has a negligible impact on the evolution of NSCs in low-mass galaxies, while it leads to their full disruption in galaxies more massive than $\sim 10^{11} M_{\odot}$. In galaxies of intermediate luminosity $\left(\sim 10^{10} M_{\odot}\right)$, MBH mergers cause the partial dissolution of the clusters. Such partially eroded clusters appear at $z=0$ significantly underweight relative to linear NSC-host spheroid scaling correlations. The presence of an undermassive NSC population results in an increased scatter of the NSC-host galaxy empirical correlations at high galaxy luminosities, for which we find evidence in observational data.

(4) We identify $M_{\mathrm{MBH}} \approx 10^{8} M_{\odot}$ as the critical value of $\mathrm{MBH}$ mass above which NSCs are significantly affected. Once the galactic $\mathrm{MBH}$ mass grows above this value, $\mathrm{MBH}$ binary mergers become highly disruptive for the central NSC, leading to the formation of a central low-density core. For galaxies more massive than the Milky Way, after such a low-density core forms, the central galactic regions are likely to remain depleted of stars throughout the subsequent evolution of the galaxy.

(5) Our models predict that the fraction of nucleated earlytype galaxies containing an accreting $\mathrm{MBH}$ (with bolometric luminosity $L>10^{10} L_{\odot}$ ) increases from $5 \%$ at $M_{\mathrm{gx}} \approx 10^{9} M_{\odot}$ to $30 \%$ at $M_{\mathrm{gx}} \approx 10^{11} M_{\odot}$. These fractions are found to be much smaller for late-type galaxies, for which only $\sim 10 \%$ of nucleated galaxies with $M_{\mathrm{gx}} \approx 10^{11} M_{\odot}$ also contain an active $\mathrm{MBH}$. Among galaxies of all types, the fraction of mixed systems containing both an $\mathrm{MBH}$ and an NSC is $\sim 20 \%$ at $10^{9} M_{\odot} \lesssim M_{\mathrm{gx}} \lesssim 10^{11} M_{\odot}$.

(6) We found that the results shown in this paper are quite robust when we change the cluster formation efficiency, $f_{\mathrm{gc}} \lesssim 0.2$; when we allow $f_{\mathrm{gc}}$ to vary with galactic properties 
(e.g., we set it to $0.07,0.04$, and 0.5 in disk, quiescent, and starburst galaxies, respectively; Kruijssen 2012); when we change the MBH seed model (i.e., light-seed models vs. heavy-seed models, with several halo occupation numbers at high redshift); and when we change other details of our model, such as merger-tree resolution, initial redshift of the simulations, prescriptions for AGN feedback, etc.

In conclusion, our study supports a scenario in which black holes form inside NSCs with a low mass fraction, so that NSCs are initially the dominant central component of galaxies. After their formation, NSCs and MBHs grow in parallel on their own scaling correlations with host galaxy properties, with NSCs dominating in low-mass spheroids and $\mathrm{MBHs}$ dominating in high-mass galaxies. The simple fact that NSCs and MBHs grow on different scaling correlations explains the well-known transition from $\mathrm{MBH}$ - to NSC-dominated galaxies as one proceeds from dwarfs to giant ellipticals, without the need of invoking competitive feedback processes from young NSCs and/or AGN activity.

During the course of this work, we have benefited from conversations with several colleagues, including M. Colpi, D. Merritt, N. Murray, and A. Sesana. We acknowledge support from a CIERA postdoctoral fellowship at Northwestern University (to F.A.); from the European Union's Seventh Framework Programme (FP7/PEOPLE-2011-CIG) through the Marie Curie Career Integration Grant GALFORMBHS PCIG11-GA-2012-321608 (to E.B.); from ERC project 267117 (DARK) hosted by Université Pierre et Marie Curie -Paris 6; and at JHU by National Science Foundation grant OIA-1124403 (to J.S.). F.A. acknowledges hospitality from the Institut d'Astrophysique de Paris, where the early plan for this work was conceived. E.B. and J.S. acknowledge hospitality from the Lorentz Center (Leiden, NL), where part of this work was carried out. Computations were performed on the gpc supercomputer at the SciNet HPC Consortium, as well as on the Horizon Cluster at the Institut d'Astrophysique de Paris.

\section{REFERENCES}

Agarwal, M., \& Milosavljević, M. 2011, ApJ, 729, 35

Aharon, D., \& Perets, H. B. 2015, ApJ, 799, 185

Antonini, F. 2013, ApJ, 763, 62

Antonini, F. 2014, ApJ, 794, 106

Antonini, F., Barausse, E., \& Silk, J. 2015, ApJL, 806, L8

Antonini, F., Capuzzo-Dolcetta, R., Mastrobuono-Battisti, A., \& Merritt, D. 2012, ApJ, 750, 111

Arca-Sedda, M., \& Capuzzo-Dolcetta, R. 2014, MNRAS, 444, 3738

Balcells, M., Graham, A. W., Domínguez-Palmero, L., \& Peletier, R. F. 2003, ApJL, 582, L79

Balcells, M., Graham, A. W., \& Peletier, R. F. 2007, ApJ, 665, 1084 Baldassare, V. F., Gallo, E., Miller, B. P., et al. 2014, ApJ, 791, 133 Barausse, E. 2012, MNRAS, 423, 2533 ; Erratum 2014, MNRAS, 440, 1295 Begelman, M. C., Blandford, R. D., \& Rees, M. J. 1980, Natur, 287, 307 Begelman, M. C., Volonteri, M., \& Rees, M. J. 2006, MNRAS, 370, 289

Bekki, K., Couch, W. J., Drinkwater, M. J., \& Shioya, Y. 2004, ApJL, 610, L13

Bekki, K., \& Graham, A. 2010, ApJL, 714, L313

Bell, E. F., \& de Jong, R. S. 2001, ApJ, 550, 212

Bell, E. F., McIntosh, D. H., Katz, N., \& Weinberg, M. D. 2003, ApJS, 149,289

Bender, R., Kormendy, J., Bower, G., et al. 2005, ApJ, 631, 280

Bernardi, M., Sheth, R. K., Annis, J., et al. 2003, AJ, 125, 1849

Bigiel, F., Leroy, A., Walter, F., et al. 2010, AJ, 140, 1194

Bik, A., Lamers, H. J. G. L. M., Bastian, N., Panagia, N., \& Romaniello, M. 2003, A\&A, 397, 473
Binney, J., \& Tremaine, S. 1987, in Galactic Dynamics (Princeton, NJ: Princeton Univ. Press), 747

Böker, T., Laine, S., van der Marel, R. P., et al. 2002, AJ, 123, 1389

Böker, T., Sarzi, M., McLaughlin, D. E., et al. 2004, AJ, 127, 105

Bolatto, A. D., Leroy, A. K., Jameson, K., et al. 2011, ApJ, 741, 12

Boylan-Kolchin, M., Ma, C.-P., \& Quataert, E. 2008, MNRAS, 383, 93

Campanelli, M., Lousto, C. O., Zlochower, Y., \& Merritt, D. 2007, PhRvL, 98, 231102

Capuzzo-Dolcetta, R., \& Miocchi, P. 2008, MNRAS, 388, L69

Carollo, C. M., Stiavelli, M., \& Mack, J. 1998, AJ, 116, 68

Carson, D. J., Barth, A. J., Seth, A. C., et al. 2015, arXiv:1501.05586

Cleveland, W. 1979, Am. Stat., 74, 82936

Cleveland, W., \& Devlin, S. J. 1988, Am. Stat., 83, 596

Colpi, M. 2014, SSRv, 183, 189

Côté, P., Piatek, S., Ferrarese, L., et al. 2006, ApJS, 165, 57

Craven, P., \& Wahba, G. 1979, NuMat, 31, 377

de Grijs, R., Anders, P., Bastian, N., et al. 2003, MNRAS, 343, 1285

Dehnen, W. 1993, MNRAS, 265, 250

den Brok, M., Peletier, R. F., Seth, A., et al. 2014, MNRAS, 445, 2385

Dekel, A., \& Cox, T. J. 2006, MNRAS, 370, 1445

De Lorenzi, F., Hartmann, M., Debattista, V. P., Seth, A. C., \& Gerhard, O. 2013, MNRAS, 429, 2974

Duschl, W. J., Strittmatter, P. A., \& Biermann, P. L. 2000, A\&A, 357, 1123

Dutton, A. A., \& van den Bosch, F. C. 2009, MNRAS, 396, 141

Dye, S., Furlanetto, C., Swinbank, A. M., et al. 2015, arXiv:1503.08720

Erwin, P., \& Gadotti, D. A. 2012, AdAst, 2012, 946368

Fakhouri, O., Ma, C.-P., \& Boylan-Kolchin, M. 2010, MNRAS, 406, 2267

Feldmeier, A., Neumayer, N., Seth, A., et al. 2014, A\&A, 570, A2

Ferrarese, L. 2002, ApJ, 578, 90

Ferrarese, L., Côté, P., Dalla Bontà, E., et al. 2006, ApJL, 644, L21

Ferrarese, L., \& Ford, H. 2005, SSRv, 116, 523

Fox, J. 1999, Nonparametric Regression Analysis, Typescript, McMaster University

Frank, J., King, A., \& Raine, D. J. 2002, Accretion Power in Astrophysics (Cambridge, UK: Cambridge Univ. Press)

Gebhardt, K., Lauer, T. R., Kormendy, J., et al. 2001, AJ, 122, 2469

Genzel, R., Tacconi, L. J., Lutz, D., et al. 2015, ApJ, 800, 20

Georgiev, I. Y., \& Böker, T. 2014, MNRAS, 441, 3570

Ghez, A. M., Klein, B. L., Morris, M., \& Becklin, E. E. 1998, ApJ, 509, 678 Gieles, M., \& Baumgardt, H. 2008, MNRAS, 389, L28

Gillessen, S., Eisenhauer, F., Trippe, S., et al. 2009, ApJ, 692, 1075

Gnedin, O. Y., Ostriker, J. P., \& Tremaine, S. 2014, ApJ, 785, 71

Golub, G., Heath, M., \& Wahba, G. 1979, Technometrics, 21, 215

González Delgado, R. M., Pérez, E., Cid Fernandes, R., \& Schmitt, H. 2008, AJ, 135, 747

Graham, A. W. 2012, MNRAS, 422, 1586

Graham, A. W., \& Driver, S. P. 2007, ApJ, 655, 77

Graham, A. W., \& Guzmán, R. 2003, AJ, 125, 2936

Graham, A. W., \& Spitler, L. R. 2009, MNRAS, 397, 2148

Granato, G. L., De Zotti, G., Silva, L., Bressan, A., \& Danese, L. 2004, ApJ, 600,580

Gualandris, A., \& Merritt, D. 2008, ApJ, 678, 780

Gullieuszik, M., Greggio, L., Falomo, R., Schreiber, L., \& Uslenghi, M. 2014, A\&A, 568, A89

Gültekin, K., Richstone, D. O., Gebhardt, K., et al. 2009, ApJ, 698, 198

Haehnelt, M. G., \& Kauffmann, G. 2002, MNRAS, 336, L61

Haiman, Z., Ciotti, L., \& Ostriker, J. P. 2004, ApJ, 606, 763

Haiman, Z., Kocsis, B., \& Menou, K. 2009, ApJ, 700, 1952

Häring, N., \& Rix, H.-W. 2004, ApJL, 604, L89

Harris, W. 1996, AJ, 112, 1487

Hartmann, M., Debattista, V. P., Seth, A., Cappellari, M., \& Quinn, T. R. 2011, MNRAS, 418, 2697

Hoffman, L., \& Loeb, A. 2007, MNRAS, 377, 957

Holley-Bockelmann, K., \& Khan, F. M. 2015, arXiv:1505.06203

Kawakatu, N., \& Umemura, M. 2002, MNRAS, 329, 572

Kawakatu, N., Umemura, M., \& Mori, M. 2003, ApJ, 583, 85

Kennicutt, R. C., Jr. 1998, ApJ, 498, 541

Kennicutt, R. C., \& Evans, N. J. 2012, ARA\&A, 50, 531

Khan, F. M., Just, A., \& Merritt, D. 2011, ApJ, 732, 89

King, I. R. 1962, AJ, 67, 471

Kormendy, J., \& Ho, L. C. 2013, ARA\&A, 51, 511

Koushiappas, S. M., Bullock, J. S., \& Dekel, A. 2004, MNRAS, 354, 292

Kregel, M., van der Kruit, P. C., \& Freeman, K. C. 2005, MNRAS, 358, 503

Kruijssen, J. M. D. 2012, MNRAS, 426, 3008 
Kruijssen, J. M. D., Longmore, S. N., Elmegreen, B. G., et al. 2014, MNRAS, 440,3370

Krumholz, M. R. 2012, ApJ, 759, 9

Krumholz, M. R., McKee, C. F., \& Tumlinson, J. 2009, ApJ, 699, 850

Lapi, A., Raimundo, S., Aversa, R., et al. 2014, ApJ, 782, 69

Lauer, T. R., Bender, R., Kormendy, J., Rosenfield, P., \& Green, R. F. 2012, ApJ, 745, 121

Leigh, N., Böker, T., \& Knigge, C. 2012, MNRAS, 424, 2130

Leigh, N. W. C., Georgiev, I. Y., Böker, T., Knigge, C., \& den Brok, M. 2015, MNRAS, 451, 5378

Leroy, A. K., Bolatto, A. D., Ostriker, E. C., et al. 2015, ApJ, 801, 25

Li, K. C. 1985, AnSta, 13, 1352

Lodato, G., \& Natarajan, P. 2006, MNRAS, 371, 1813

Madau, P., Haardt, F., \& Dotti, M. 2014, ApJL, 784, L38

Madau, P., \& Rees, M. J. 2001, ApJL, 551, L27

Magorrian, J., Tremaine, S., Richstone, D., et al. 1998, AJ, 115, 2285

Matthews, L. D., Gallagher, J. S., III, Krist, J. E., et al. 1999, AJ, 118, 208

McLaughlin, D. E., King, A. R., \& Nayakshin, S. 2006, ApJL, 650, L37

Merritt, D. 2006, ApJ, 648, 976

Merritt, D. 2013, Dynamics and Evolution of Galactic Nuclei (Princeton, NJ: Princeton Univ. Press)

Merritt, D., \& Cruz, F. 2001, ApJL, 551, L41

Merritt, D., Ferrarese, L., \& Joseph, C. L. 2001, Sci, 293, 1116

Merritt, D., Piatek, S., Portegies Zwart, S., \& Hemsendorf, M. 2004, ApJL, 608, L25

Merritt, D., Schnittman, J. D., \& Komossa, S. 2009, ApJ, 699, 1690

Merritt, D., \& Szell, A. 2006, ApJ, 648, 890

Miller, M. C., \& Davies, M. B. 2012, ApJ, 755, 81

Milosavljević, M. 2004, ApJL, 605, L13

Milosavljević, M., \& Merritt, D. 2001, ApJ, 563, 34

Neumayer, N., \& Walcher, C. J. 2012, AdAst, 2012, 709038

Parkinson, H., Cole, S., \& Helly, J. 2008, MNRAS, 383, 557

Perets, H. B., \& Mastrobuono-Battisti, A. 2014, ApJL, 784, L44
Pfuhl, O., Fritz, T. K., Zilka, M., et al. 2011, ApJ, 741, 108

Phillips, A. C., Illingworth, G. D., MacKenty, J. W., \& Franx, M. 1996, AJ, 111,1566

Rossa, J., van der Marel, R. P., Böker, T., et al. 2006, AJ, 132, 1074

Schödel, R., Feldmeier, A., Kunneriath, D., et al. 2014, A\&A, 566, A47

Scott, N., \& Graham, A. W. 2013, ApJ, 763, 76

Sesana, A., Barausse, E., Dotti, M., \& Rossi, E. M. 2014, ApJ, 794, 104

Sesana, A., \& Khan, F. M. 2015, arXiv:1505.02062

Seth, A., Agüeros, M., Lee, D., \& Basu-Zych, A. 2008a, ApJ, 678, 116

Seth, A. C., Blum, R. D., Bastian, N., Caldwell, N., \& Debattista, V. P. 2008b, ApJ, 687, 997

Seth, A. C., Dalcanton, J. J., Hodge, P. W., \& Debattista, V. P. 2006, AJ, 132,2539

Shen, S., Mo, H. J., White, S. D. M., et al. 2003, MNRAS, 343, 978

Swinbank, M., Dye, S., Nightgale, J., et al. 2015, arXiv:1505.05148

Taffoni, G., Mayer, L., Colpi, M., \& Governato, F. 2003, MNRAS, 341, 434

Tremaine, S., Gebhardt, K., Bender, R., et al. 2002, ApJ, 574, 740

Tremaine, S. D., Ostriker, J. P., \& Spitzer, L., Jr. 1975, ApJ, 196, 407

Turner, M. L., Côté, P., Ferrarese, L., et al. 2012, ApJS, 203, 5

Umemura, M. 2001, ApJL, 560, L29

van den Bergh, S. 1986, AJ, 91, 271

van Meter, J. R., Miller, M. C., Baker, J. G., Boggs, W. D., \& Kelly, B. J. 2010, ApJ, 719, 1427

Vasiliev, E. 2014, arXiv:1411.1762

Vasiliev, E., Antonini, F., \& Merritt, D. 2014, ApJ, 785, 163

Vasiliev, E., Antonini, F., \& Merritt, D. 2015, arXiv:1505.05480

Volonteri, M., Lodato, G., \& Natarajan, P. 2008, MNRAS, 383, 1079

Walcher, C. J., Böker, T., Charlot, S., et al. 2006, ApJ, 649, 692

Walcher, C. J., van der Marel, R. P., McLaughlin, D., et al. 2005, ApJ, 618, 237

Wehner, E. H., \& Harris, W. E. 2006, ApJL, 644, L17

Yu, Q. 2002, MNRAS, 331, 935

Yusef-Zadeh, F., Bushouse, H., \& Wardle, M. 2012, ApJ, 744, 24 